\title{
How Physics Defines the LHC Environment and Detectors
}

\author{
Dan Green \\ Fermilab
}

\section{Introduction}

Much has been written [1] about the general purpose experiments, ATLAS and CMS, which have recently been completed and are installed in the Large Hadron Collider (LHC) at the CERN laboratory. The accelerator itself has a URL [2] and a comprehensive Technical Design Report (TDR) [3].

The experiments themselves have home pages, [4], [5], which are sources of considerable public information. In particular, the physics capabilities of the detectors are made available there. In addition the technical details of the experiments have recently been written up as a reference source for specifying the detectors [6]. The ATLAS and CMS experiments have together also been described and compared in a review article, [7] which gives many detailed descriptions of the detector performance.

Given this wealth of information there yet remains a need to explain why the search for new physics beyond the Standard Model (SM) defines the requirements for detectors at a proton-proton collider and why specific choices were made by the ATLAS and CMS experimenters in order to meet those requirements. Indeed, the "why" of these detectors is the main subject of this volume. This introductory chapter explores the environment in which the detectors must operate and allow the extraction of the "new physics" that the LHC will make accessible.

\section{Electroweak Symmetry Breaking (EWSB)}

It has been known for more than twenty years that the electroweak bosons exist and are massive, with the $\mathrm{Z}$ having $91.2 \mathrm{GeV}$ of mass while the $\mathrm{W}$ has $80.4 \mathrm{GeV}$. Since these masses imply a breaking of the electroweak gauge symmetry, an explanation which extends beyond the SM is needed. The necessary energy scale to be explored is set by looking at a process such as $e^{+}+e^{-} \rightarrow W^{+}+W^{-}$. In the SM this electroweak process has an $S$ wave amplitude, $A_{0}$, which violates perturbative unitarity at high center of momentum, C.M., energies $\sqrt{s}$ :

$$
\begin{aligned}
& A_{o}=\alpha_{W} s / 16 M_{W}^{2}<1 \\
& \alpha_{W}=\alpha / \sin ^{2} \theta_{W} \sim 1 / 31.6
\end{aligned}
$$

Therefore, new physics must intervene at or before the "terascale" where $\sqrt{s}<4 M_{W} / \sqrt{\alpha_{W}}=1.8 \mathrm{TeV}$. The angle $\theta_{W}$ is the Weinberg angle and $\alpha_{W}$ is the 
electroweak coupling constant while $\alpha$ is the electromagnetic fine structure constant [8]. Units with $\hbar=c=1$ are used in all the equations.

Another indication of the appropriate energy scale for new physics is set by considering a weakly interacting, neutral, stable particle which would make up the "dark matter" which has been inferred in cosmological and astrophysical studies. If such a particle had a mass of order $1 \mathrm{TeV}$ it would then have the correct relic density to be a candidate particle for dark matter. It is to be hoped that this fact is not simply coincidental [9].

The historical approach to this high energy scale is shown in Fig. 1 where the available energy to make new particles is plotted as a function of the year when a new accelerator facility came on line. The exponential growth of available C.M. energy with time has clearly slowed lately. It is also clear that the new LHC accelerator, with a C.M. energy of $14 \mathrm{TeV}$ is the only facility now available which can decisively probe the necessary terascale. The C.M. energy of the scattering of the fundamental constituents is less that the proton-proton C.M. energy because the protons are composites composed of quarks and gluons.

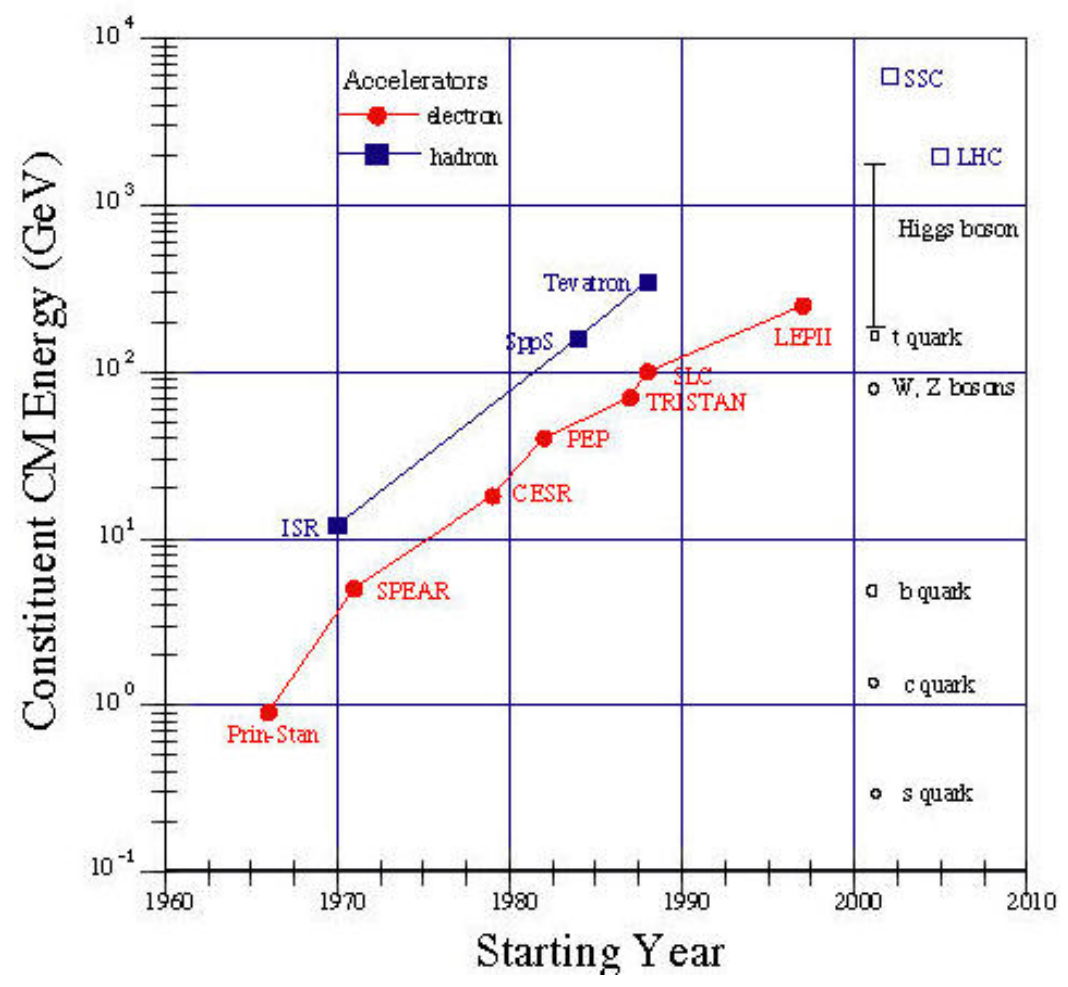

Figure 1: The available C.M. energy at recent accelerator facilities as a function of the starting year of the facility. The first data taking run at the LHC will be in 2009 . The masses of the quarks and bosons are also indicated and the possible range of the Higgs mass. 
It should be noted that the simplest extension of the SM is to posit a scalar particle which couples to mass, the Higgs boson, which then has a vacuum expectation value which breaks the electroweak symmetry, couples to the gauge bosons and gives them a prescribed mass [10]. Further postulating a Higgs Yukawa coupling to fermions, albeit with undetermined coupling strength, allows for a SM extension which compactly gives mass to all SM particles. Nevertheless, this is only the simplest SM extension and remains a hypothesis until the terascale is fully explored at the LHC and the "Higgs boson" is either discovered or not.

\section{LHC Luminosity and Energy}

Given the importance of the terascale, and the breakdown of electroweak vector boson scattering at that scale, a requirement of the LHC is to enable the study of vector boson scattering at a C.M. energy $1 \mathrm{TeV}$. Exploring the dynamics of pairs of vector bosons with a pair mass $\sim 1 \mathrm{TeV}$ would also probe the triple and quartic interactions of the gauge bosons at the desired mass scale.

The vector bosons decay to either quark or lepton pairs. However, the enormous backgrounds which exist at the LHC due to strongly produced QCD processes make the detection of leptonic decays experimentally favored. This fact explains why LHC detectors tend to focus on lepton detection. The branching ratio for a $\mathrm{W}$ to decay to a muon plus neutrino is, $B_{\mu} \sim 1 / 9$. A crude estimate of the cross section for electroweak $\mathrm{W}+\mathrm{W}$ production at the LHC with subsequent $\mathrm{W}$ decay to muons is:

$$
\sigma\left(p+p \rightarrow W^{+}+W^{-} \rightarrow \mu^{+}+v_{\mu}+\mu^{-}+\bar{v}_{\mu}\right) \sim\left(\alpha_{W}^{2} / \hat{s}\right) B_{\mu}^{2}
$$

This estimate gives a 5 fb cross section times branching ratio squared for a $\mathrm{W}$ pair mass of $\sqrt{\hat{s}}=1 \mathrm{TeV}$.

A detailed Monte Carlo model [11] result for $W+W$ pair production is shown in Fig. 2. The cross section for $W+W$ mass above $1 \mathrm{TeV}$ is $640 \mathrm{fb}$. Requiring two muons in the final state yields a cross section of $7.9 \mathrm{fb}$ in reasonable agreement with the previous estimate. In order to have sufficient statistical power in studying this process, the LHC should provide $100 \mathrm{fb}^{-1} /$ year. Taking a running time, $\mathrm{T}, \mathrm{of} 1 \mathrm{10}^{7}$ sec/yr ( $\sim 30 \%$ of the calendar year) there will be $\sim 790 \mathrm{~W}+\mathrm{W}$ events produced per year with a mass above $1 \mathrm{TeV}$ which decay into the experimentally favorable final state containing two muons. A similar event sample will be available in the two electron final state and twice that in the muon plus electron final state.

Therefore, the LHC was required both to have a C.M. energy of $14 \mathrm{TeV}$ to reach and explore well the terascale where a mechanism for EWSB must be found and it must have a luminosity, $L$, of: 


$$
\begin{aligned}
& L=10^{34} / \mathrm{cm}^{2} \text { sec, } T=10^{7} \mathrm{sec} \\
& L T=10^{41} / \mathrm{cm}^{2} y r=100 \mathrm{fb}^{-1} / \mathrm{yr}
\end{aligned}
$$

Note that the LHC energy is seven times that of the existing Fermilab Tevatron and the full design luminosity is about thirty times larger. Thus the LHC represents a major improvement in discovery potential in the study of EWSB over the presently operating hadron collider facility.

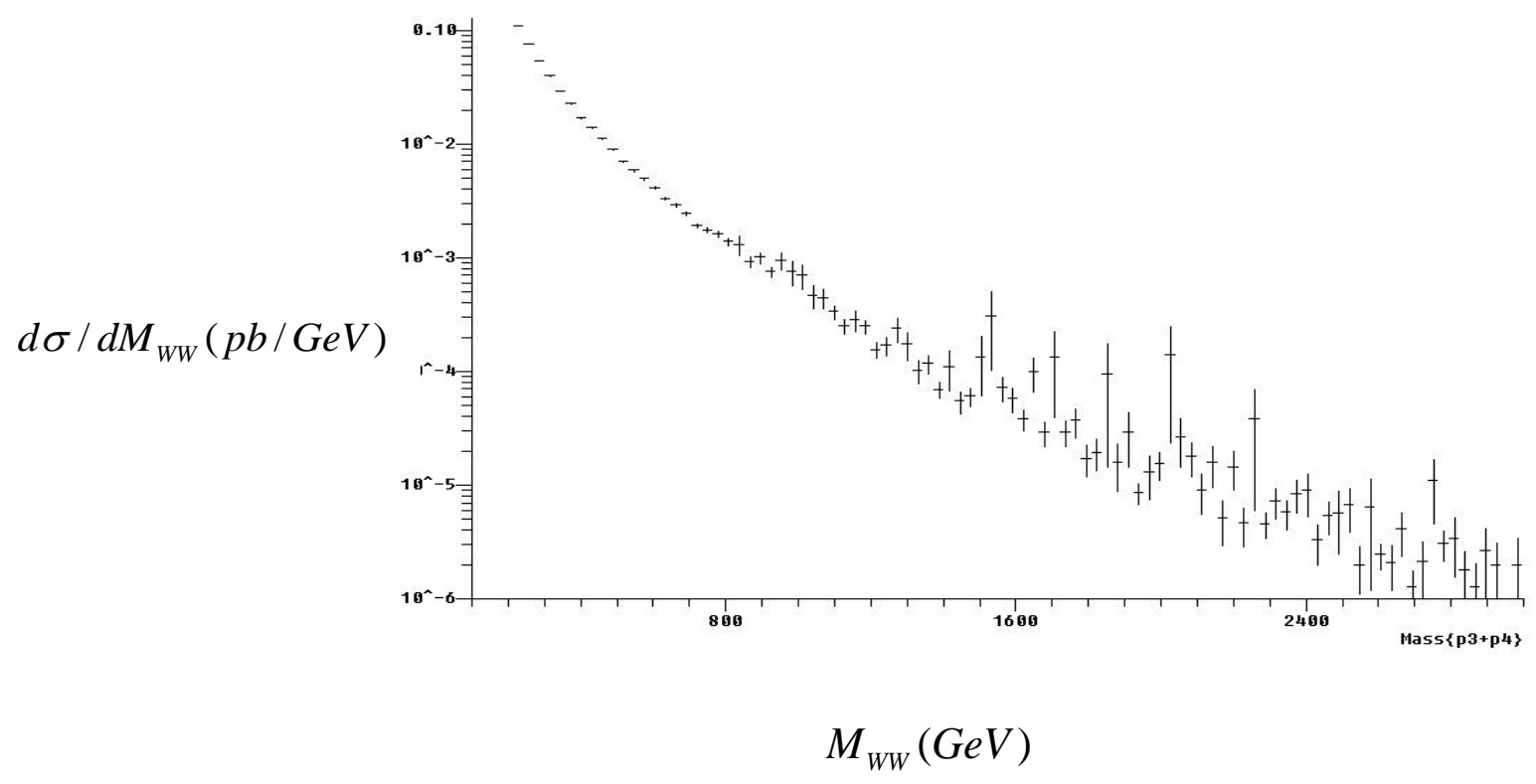

Figure 2: COMPHEP [11] calculation for the cross section for the production of $W$ pairs as a function of the pair mass at C.M. energy of $14 \mathrm{TeV}$ in $\mathrm{p}+\mathrm{p}$ collisions.

\section{Global Detector Properties}

The high luminosity which is required of the LHC by our need to explore terascale physics means that the detectors will be exposed to high particle rates. Therefore LHC experiments will require fast, radiation hard and finely segmented detectors. The speed of the detectors sets a scale for the accelerator radiofrequency, r.f., bunch structure. It is assumed in what follows that all the detectors can be operated at a speed which can resolve the time between two successive r.f. bunches which is 25 nsec at the LHC.

Consider, for example, a silicon solid state detector operated with a bias voltage which creates an electric field, E. The charge collection time is, $\tau=d / \mu E$, where $\mathbf{d}$ is the thickness of the detector and $\mu$ is the hole or electron mobility. Numerically, for $d=300 \mu \mathrm{m}$, the electron drift velocity at a typical depletion voltage of $\sim 50 \mathbf{V}=\mathbf{d E}$ is $\mu E \sim 42 \mu \mathrm{m} / \mathrm{n}$ sec leading to a charge collection time of $\sim 7 \mathrm{nsec}$. The holes are $\sim 3$ 
times slower, so a charge collection of 25 nsec is well matched to the LHC bunch crossing spacing. Shorter bunch crossing times are not useful because the detectors would simply be forced to integrate over the multiple bunch crossings occurring within their resolving time.

Having progressed from the need to study EWSB decisively to the C.M. energy and luminosity of the LHC, a "generic" detector will now be considered. This detector is defined simply to serve as a mechanism whereby general features of LHC detectors can be explored while detailed detector concepts and technological choices are deferred to later chapters of the text.

Some of the relevant cross sections as a function of C.M. energy are shown in Fig. 3.

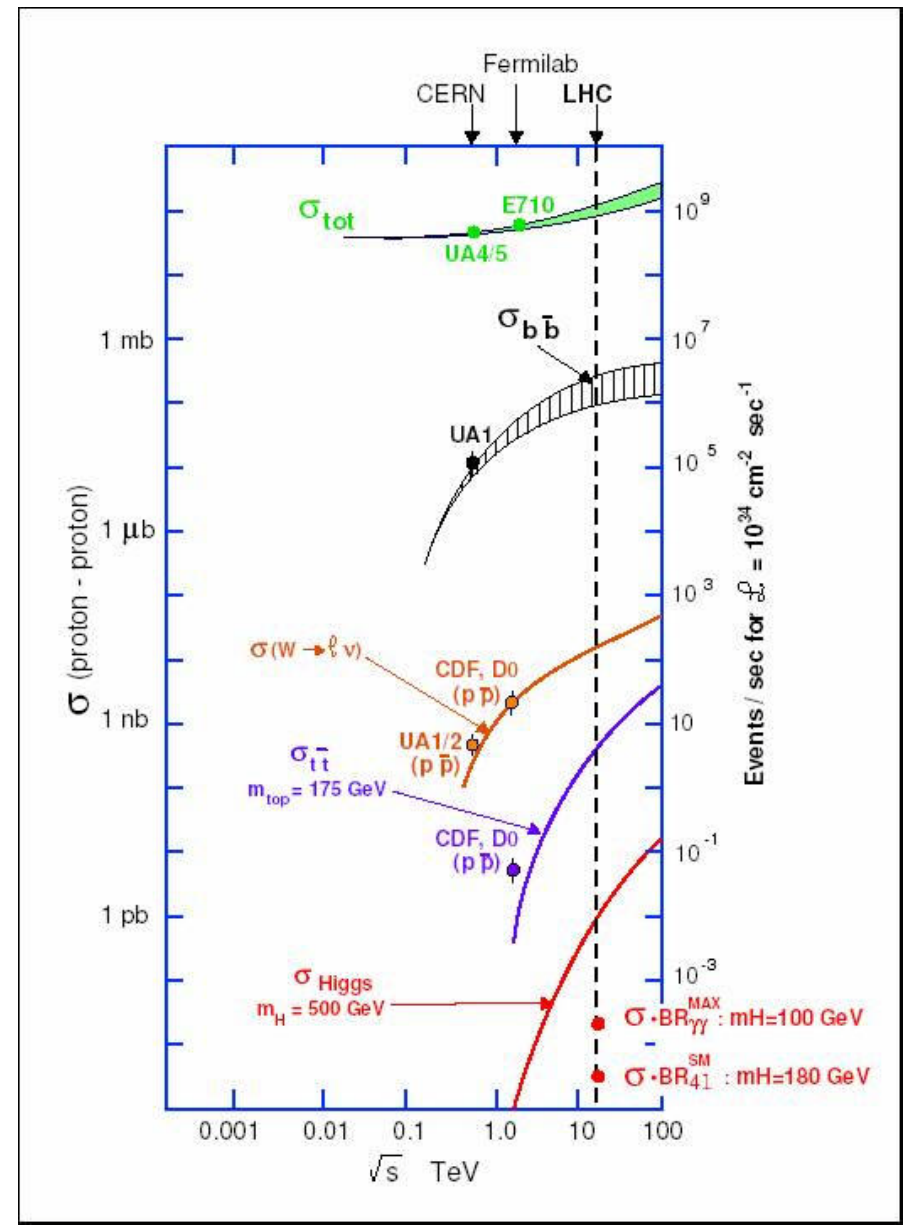

Figure 3: Cross sections as a function of C.M. energy for hadron colliders. Note that the cross section scales shown cover more that twelve orders of magnitude.

The scale shown covers many orders of magnitude which reinforces the fact that the LHC experimenters must explore rare processes in their study of the terascale. Indeed, the clean and background free detection of the rarely produced leptons 
implies that redundant measurements of the muons and electrons are required. For example $\mathbf{W}$ leptonic decays are indeed rare, $\sigma(W \rightarrow \mu+v) / \sigma_{\text {tot }} \sim 10^{-7}$.

Note that the total inelastic cross section is $\sim 100 \mathrm{mb}$. With a luminosity as defined above the total inelastic reaction rate $\mathbf{R}$ is, $R=\sigma L=10^{9} / \mathrm{sec}=1 \mathrm{GHz}$. Having chosen the r.f. bunch spacing to be 25 nsec, each crossing contains $\sim 25$ inelastic events at full luminosity. This leads to experimental issues but the luminosity that is required at the LHC and the size of the inelastic cross section makes a "pileup" of $N_{I}=25$ inelastic events in each bunch crossing unavoidable.

In designing the generic detector it is crucial to understand what part of phase space the produced particles occupy. Note that single particle relativisticly invariant phase space for a particle of mass $M$, momentum $\vec{P}$ and energy $E$ is:

$$
\begin{aligned}
& d^{4} P \delta\left(P^{2}-M^{2}\right)=d \vec{P} / E=\pi d y d P_{T}^{2} \\
& E=M_{T} \cosh y, M_{T}^{2}=M^{2}+P_{T}^{2} \\
& y \rightarrow \eta=-\log (\tan \theta / 2), P>>M
\end{aligned}
$$

Therefore if simple one particle phase space is followed by the produced particles in a typical inelastic reaction, they can be expected to be found uniformly distributed in rapidity, $y$. The momentum transverse to the proton beam directions is denoted by $P_{T}$. For light particles, $M / P<<1$, the rapidity y can be approximated by the pseudorapidity $\eta$.

Note that most of the produced particles are pions which have a mass of $0.14 \mathrm{GeV}$ while their mean transverse momentum at the LHC is expected to be $\sim 0.8 \mathrm{GeV}$. This estimate comes from extrapolation of data at lower energies, such as data shown in Fig. 6. Therefore, the angular variable, the pseudorapidity $\eta$, is a good approximation to $\mathrm{y}$ in most cases.

Data from hadron colliders on the pseudorapidity distribution of produced particles are shown in Fig. 4. Indeed, production data at $\eta \sim 0$ (i.e. near 90 degrees in the $\mathrm{p}^{+} \mathrm{p}$ C.M. system) display a roughly uniform distribution. Note also that the density, $D=1 / \sigma(d \sigma / d \eta)$, of particles in the constant region and the width of the constant region rise slowly with increasing C.M. energy.

A COMPHEP [11] calculation of the rapidity distribution of gluon jets is shown in Fig. 5. The kinematic limit for the $7 \mathrm{TeV}$ incident protons is $y_{p}= \pm 9.6$. Clearly, requiring that the LHC detectors detect most of the produced particles means that a rapidity coverage of $2 y_{\max } \sim 10$ is needed.

One also needs to know the energy distribution of the produced pions. The distribution of the transverse momentum of the inclusively produced particles is 
shown in Fig. 6. The scale for transverse momentum is low and also slowly increasing with C.M. energy. With this coverage the transverse energy in a collision which is lost in the region $|y|>5$ does not cause a significant loss of physics capability. Extrapolating these increases in particle rapidity density and transverse momentum to the LHC, a generic detector should cover a full rapidity range of 10 . The particle density per unit of rapidity will be about nine, six charged pions and three neutral pions under the assumption that all produced particles are pions and all charge
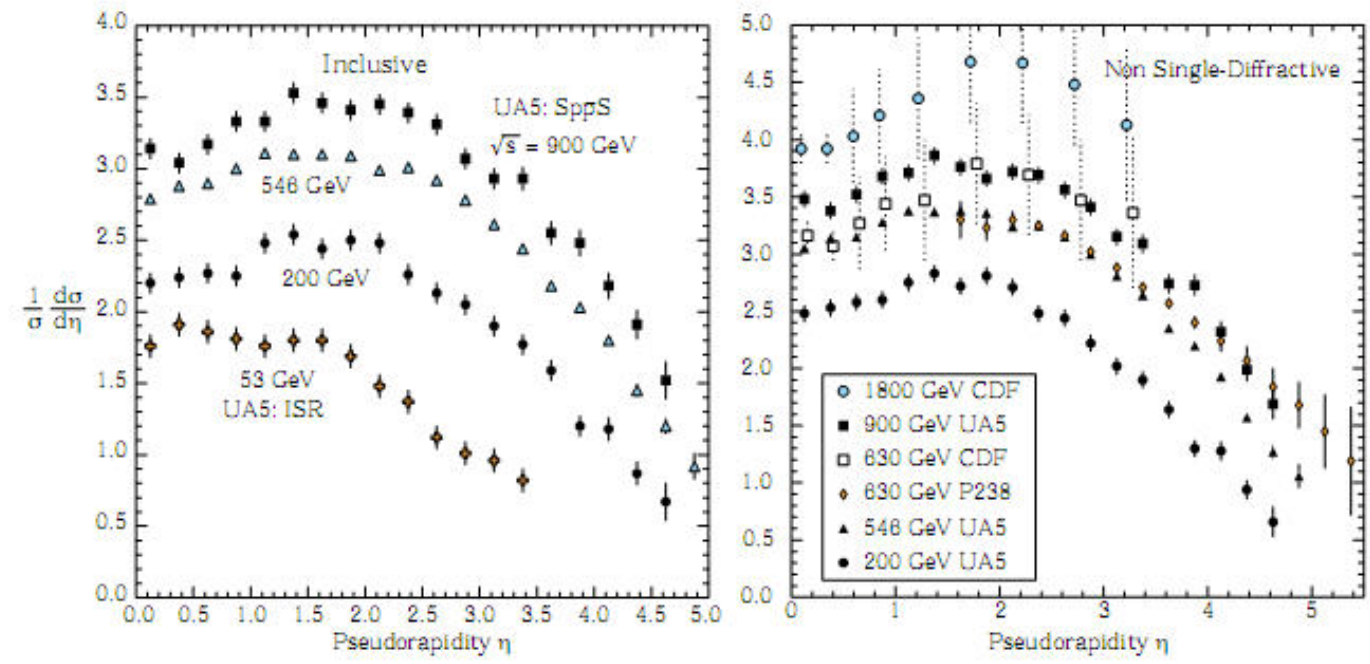

Figure 4: Distribution in pseudorapidity for produced charged particles in inelastic collisions at hadron colliders [8].

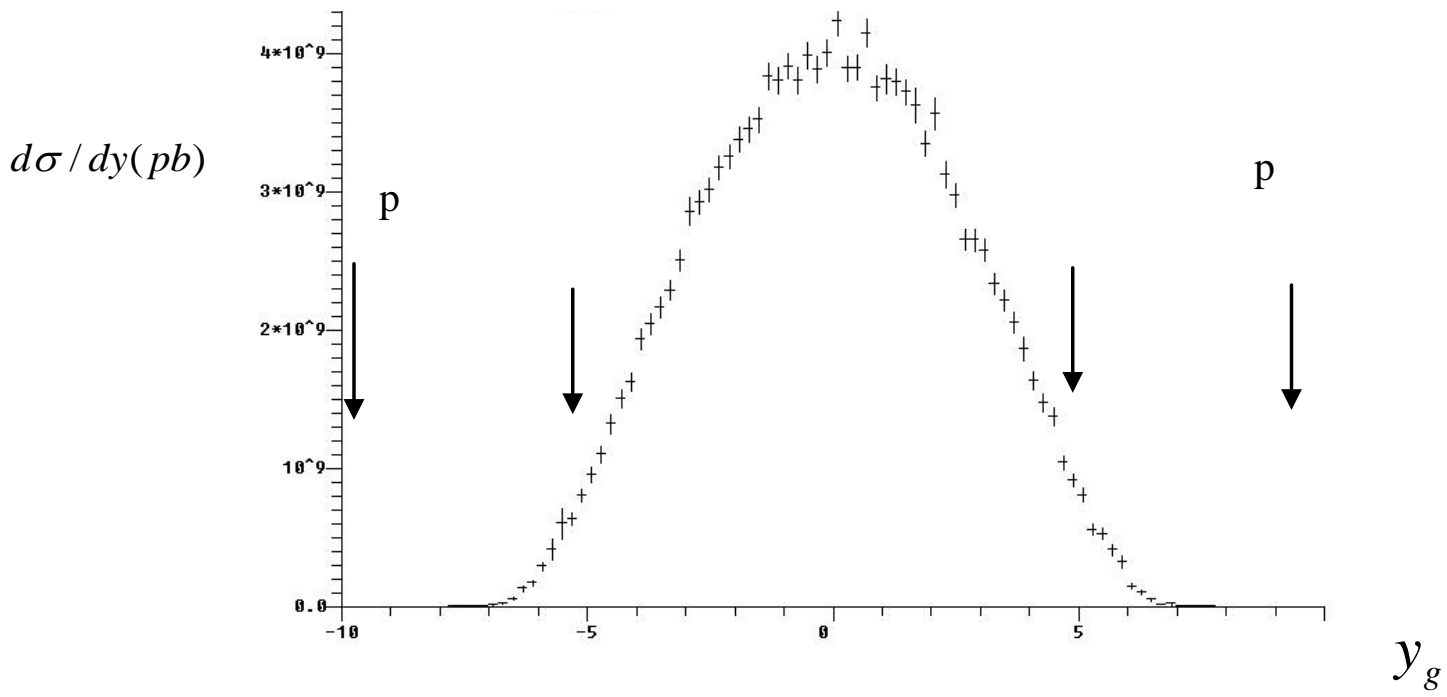

Figure 5: Rapidity distribution of low transverse momentum gluon jets at the LHC. Generic detector coverage and the incoming proton rapidities are indicated by the arrows. 
states of the pions are equally probable. The mean transverse momentum of the pions will be $\sim 0.8 \mathrm{GeV}$. Therefore in each time resolved r.f. bunch crossing there are 2250 particles in the complete detector coverage.

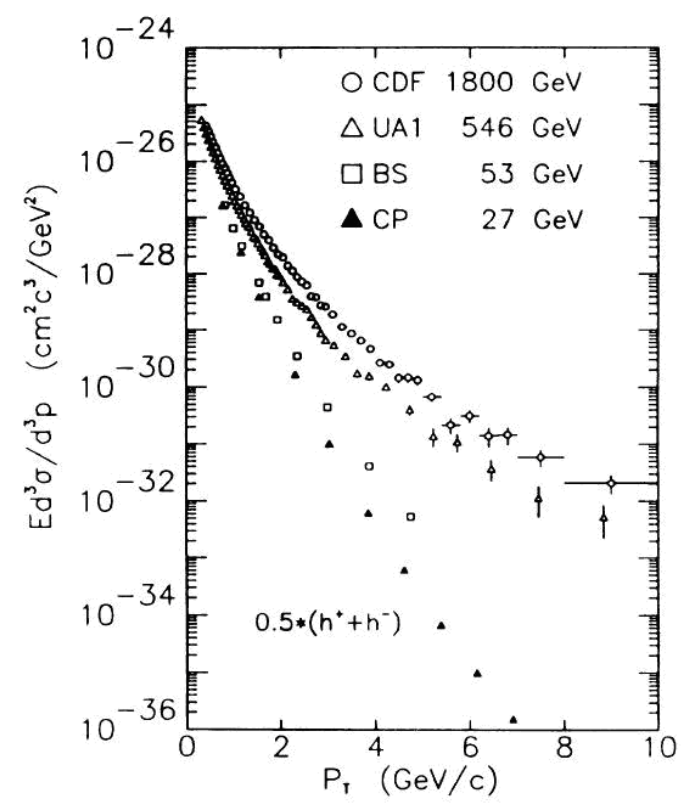

Figure 6: Transverse momentum distribution of inclusively produced charged secondary particles at hadron colliders [8].

$$
\begin{aligned}
& D \sim 9 \pi / \text { unit of } y, 6 \pi^{ \pm}+3 \pi^{o} \\
& <P_{T}>\sim 0.8 \mathrm{GeV} \\
& N_{I}\left(2 y_{\max }\right) D=2250 \text { particles, } 1.8 \mathrm{TeV} \text { total } \sum P_{T}
\end{aligned}
$$

The generic detector will see 2250 pions in each bunch crossing carrying a total of 1.8 TeV of transverse momentum. These particles are backgrounds and contribute to the "pileup" of energy which occurs within the resolving time of the detector in addition to those particles from processes which are of interest to the experimenters.

The high mass scale processes of interest populate phase space somewhat differently. For example a hypothetical $2 \mathrm{TeV}$ mass recurrence of the $\mathrm{Z}$ boson decaying into electrons preferentially appears at small rapidities as shown in Fig. 7. Therefore, the generic detector will put more stress on the low $|y|$ regions of phase space and deploy precision detectors to cover rapidities $|\mathbf{y}|<2.5$. In fact the more precise generic detectors are limited to the region $|y|<2.5$ due to their rate limitations and their ability to withstand radiation damage both to the detectors and the front end electronics. The larger $|y|$ region is covered only by radiation hardened calorimetry. 
The kinematic maximum value of rapidity for a given mass $M$ and C.M. energy can be estimated using energy and momentum conservation in the production of that mass by the incident quarks or gluons contained in the proton and moving with momentum fraction $x_{1}$ and $x_{2}$ of the proton beam which fuse together to produce mass $M$ moving with momentum fraction $\mathrm{x}$ and rapidity $\mathrm{y}$ :

$$
\begin{aligned}
& x_{1} x_{2}=M^{2} / s, x_{1}-x_{2}=x \\
& x_{1}=[M / \sqrt{s}] e^{-y} \\
& x_{2}=[M / \sqrt{s}] e^{y} \\
& y_{\max }(M) \sim \ln (\sqrt{s} / M)
\end{aligned}
$$

For the LHC, operating at a C.M. energy, $\sqrt{s}$, of $14 \mathrm{TeV}$, the maximum rapidity of $y_{\max }(2 \mathrm{TeV})=\mathbf{1 . 9}$ for a mass of $2 \mathrm{TeV}$ occurs when $x_{2}->1$ which is in rough agreement with the $y$ distribution of the leptons from the assumed two body decay of that massive particle as shown in Fig. 7. Clearly the generic detector covers this reaction with good detection efficiency. The logarithmic dependence of the maximum rapidity on C.M. energy was already seen in the pion y behavior in Fig. 4.

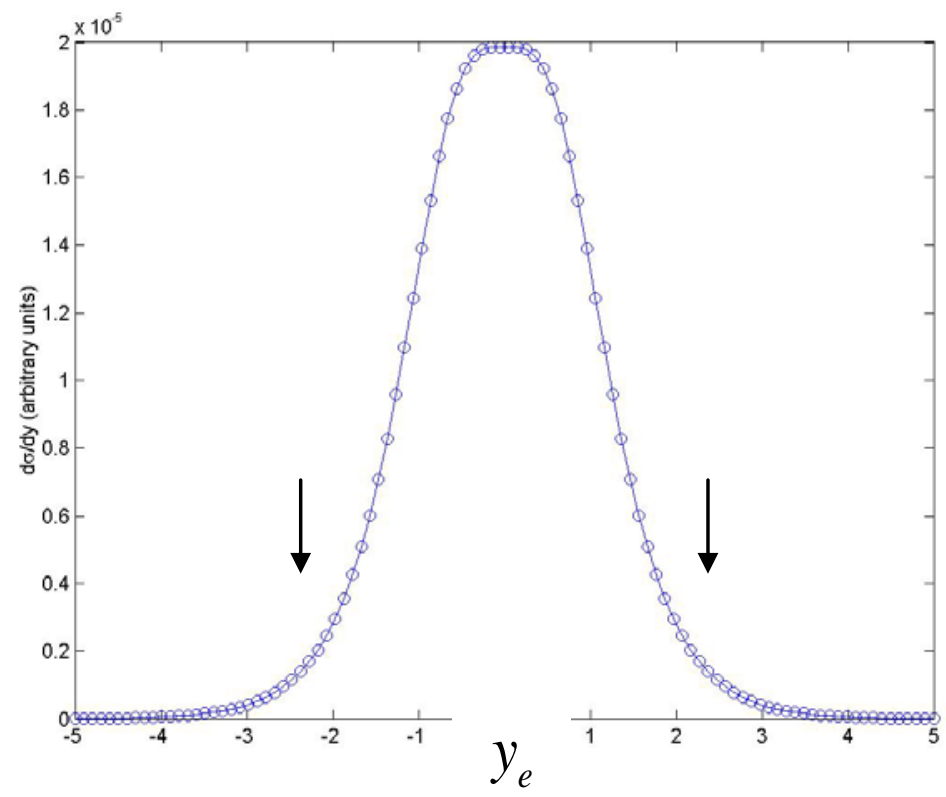

Figure 7: Rapidity distribution for the electrons resulting from the two body decay of a hypothetical $2 \mathrm{TeV}$ mass recurrence of the $\mathrm{Z}$ boson. The limit for precision detection systems at $|y|<2.5$ is indicated by the arrows.

There are also specific processes which relate to vector boson reactions which are a primary focus of LHC physics studies. Specifically, the virtual emission of a $W$ by an initial state quark in both of the protons will lead to the scattered $\mathrm{W}+\mathrm{W}$ plus 
the two quarks recoiling after $\mathrm{W}$ emission. This process is called "vector boson fusion" and is a critical component of the LHC physics program because it has a signature which allows the experimenters to tag and isolate vector boson scattering. The rapidity distribution of the final state quarks is shown in Fig. 8. Clearly, this is yet a second reason to have generic detector coverage out to \pm five units in rapidity, albeit with somewhat less precise measurement capability and no tracking.

$d \sigma / d y(p b)$

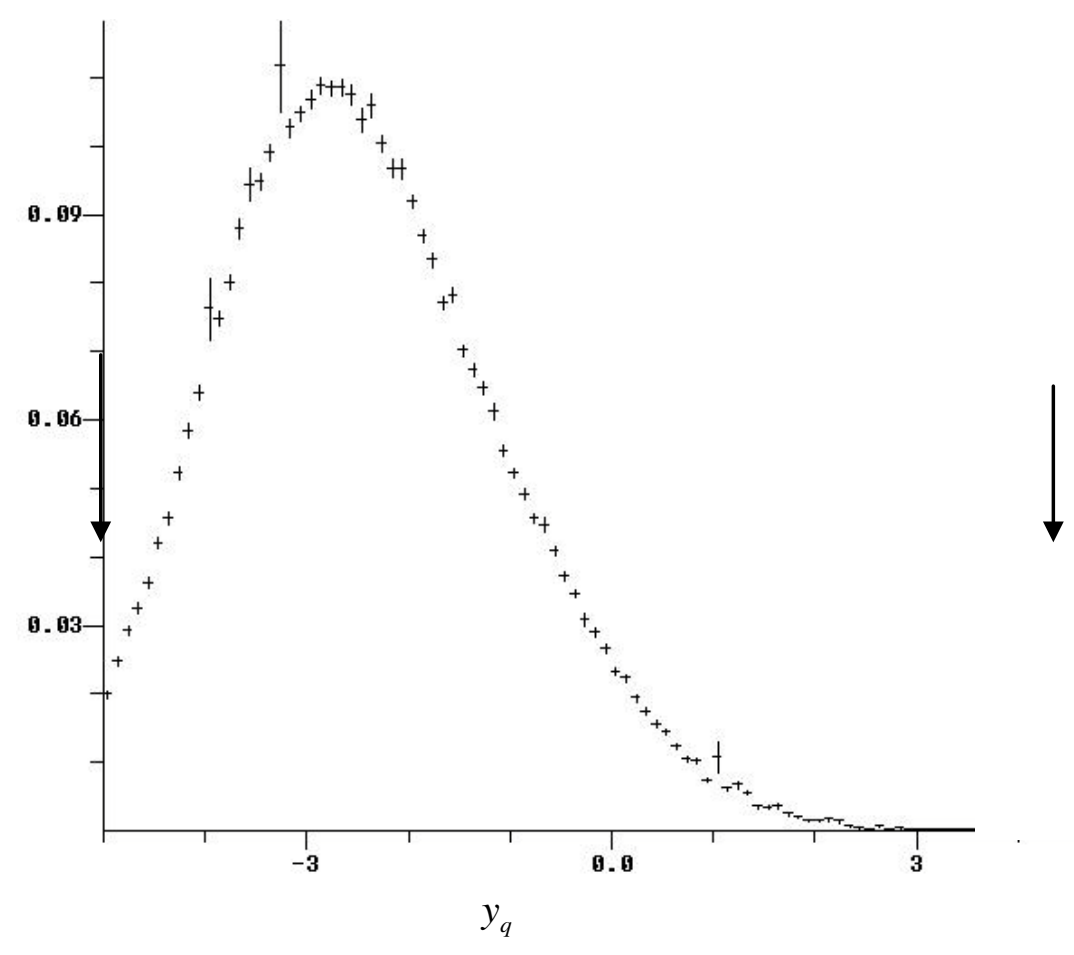

Figure 8: Rapidity distribution of the final state quark after virtual emission of a $W$ boson in the formation of a $\mathrm{W}+\mathrm{W}$ resonance of $0.2 \mathrm{TeV}$ mass via the vector boson fusion mechanism. Coverage of \pm five units of rapidity is needed for efficient detection of this process.

\section{The "Generic" Detector}

The needed full detector coverage in angle followed from the properties of inelastic events and from the vector boson fusion process. The generic detector consists of several subsystems with specific roles in particle detection and identification and is described below. The idea here is simply to observe from the physics requirements what a typical general purpose detector might look like. It is generic in the sense that specific design choices are not made but rather the general needs of the several detector subsystems are examined.

The overall coverage in rapidity of \pm 5 units follows from the distribution of inclusive inelastic secondary particle production and the desire to detect the forward going quarks from the vector boson fusion mechanism. This coverage should be "hermetic" which means that all produced particles should be detected 
and their positions and momenta should be well measured. If this is achieved, then the production of neutrinos may be inferred by vectorially adding all the observed particle transverse momenta in the final state because the initial state contains almost no transverse momentum. If an imbalance remains the existence of "missing transverse momentum" or MET implies that an undetected, non-interacting, neutral stable particle or particles has been produced which carried off the MET.

The generic detector has detection elements which are roughly of uniform extent in pseudorapidity and azimuthal angle since then they will have roughly equal probability to be struck by a secondary particle. If a detector "tower" size of,

$$
\delta \eta=\delta \phi / 2 \pi=0.1
$$

is chosen then there will be 6300 independently read out "towers" reporting a measurement of particle passage over the full coverage of the detector, $2 y_{\max }=10$. Since there are 2250 produced pions at full LHC luminosity in the detector acceptance, the mean probability for a tower to be occupied is $~ 36 \%$ (24 \% charged, $12 \%$ neutral).

The connection between the interval in polar angle and the pseudorapidity interval follows from Eq. 4. The interval at small $|\eta|$ is approximately the same for rapidity and angle.

$$
\begin{aligned}
d \eta & =\left[e^{\eta} d \theta\right] /(1+\cos \theta) \\
& \rightarrow d \theta, \eta \sim 0 \\
& \rightarrow e^{\eta} d \theta / 2=d \theta / \theta, \eta>>1
\end{aligned}
$$

The generic detector is shown in Fig. 9. There are several specific subsystems which are indicated. A general purpose detector, such as those installed at the LHC, aims to measure all the particles of the SM from each interaction as well as possible. This follows because, whatever form the physics beyond the SM takes, the final state particles will ultimately decay to the bosons, quarks, gluons, charged leptons and neutrinos of the SM. The role of the subsystems in detection and identification of the SM particles is indicated in Table 1.

The generic detector focuses on the "barrel" or wide angle region, $|\eta|<1.5$, because heavy new particles are kinematically expected to be produced there. Coverage by the vertex, tracking and electromagnetic calorimetry is limited to $|\eta|<2.5$ because of the fierce radiation field which exists at smaller angles. That field must, however, be confronted because of the high luminosity needed to explore EWSB at the terascale and the need for hermetic coverage.

The size and location of the detector subsystems follow from the physics requirements. The vertex subsystem exists to measure the secondary decay vertices 
of the heavy quarks and leptons. Therefore the vertex subsystem is as nearto the LHC vacuum beam pipe as is possible. The tracking subsystem is immersed in a

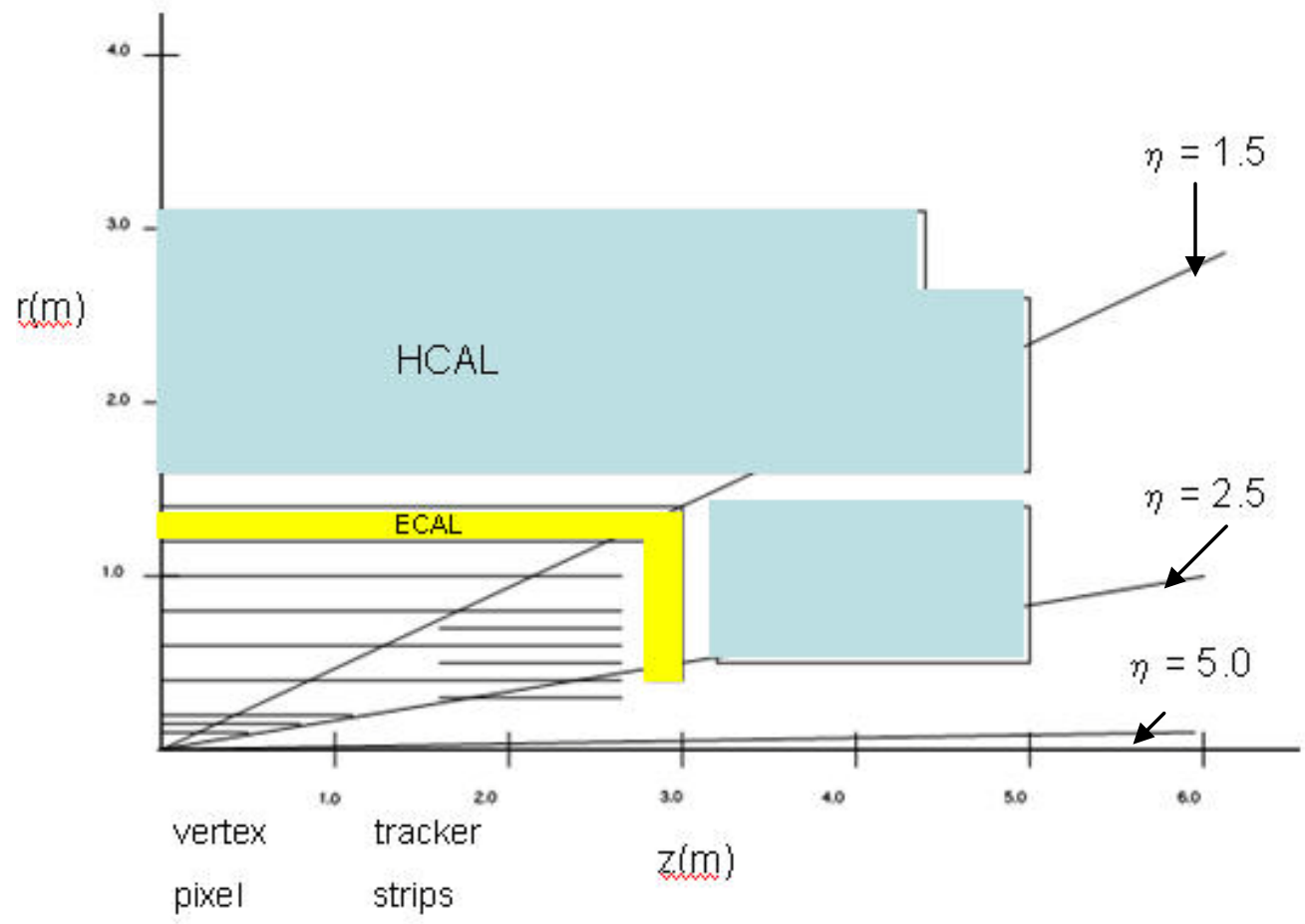

Figure 9: A “generic” LHC detector which covers \pm 5 units of pseudorapidity. Only the central \pm 2.5 units of coverage are shown here. The remaining small angle calorimetry is at $\mathrm{z}=10 \mathrm{~m}$. The muon detection system is also not shown.

Table 1

Particles of the SM and Detection And Identification in Detector Subsystems

\begin{tabular}{|c|c|c|}
\hline Particle & Signature & Generic Subsystem \\
\hline \begin{tabular}{l|l}
$u, c, t \rightarrow W+b$ & \\
$d, s, b$ & Quarks \\
$g$ &
\end{tabular} & $\begin{array}{l}\text { Jet of hadrons } \\
\left(\lambda_{o}\right)\end{array}$ & $\begin{array}{l}\text { Calorimeter } \\
\text { ECAL+HCAL }\end{array}$ \\
\hline $\mathrm{e}, \gamma$ & $\begin{array}{l}\text { Electromagnetic shower } \\
\left(X_{o}\right)\end{array}$ & $\begin{array}{l}\text { Calorimeter } \\
\text { ECAL } \\
\text { Tracker }\end{array}$ \\
\hline $\begin{array}{l}v_{e}, v_{\mu}, v_{\tau} \\
W \rightarrow \mu+v_{\mu}\end{array}$ & $\begin{array}{l}\text { Missing transverse energy } \\
(\mathrm{MET})\end{array}$ & $\begin{array}{l}\text { Calorimeter } \\
\text { ECAL+HCAL }\end{array}$ \\
\hline $\begin{array}{l}\mu, \tau \rightarrow \mu+v_{\tau}+\bar{v}_{\mu} \\
Z \rightarrow \mu+\mu\end{array}$ & $\begin{array}{l}\text { Only ionization } \\
\mathrm{dE} / \mathrm{dx}\end{array}$ & $\begin{array}{l}\text { Muon absorber and } \\
\text { detectors } \\
\text { Tracker }\end{array}$ \\
\hline $\mathrm{c}, \mathrm{b}, \tau$ & Secondary decay vertices & Vertex + Tracker \\
\hline
\end{tabular}


large solenoid (uniform field in the $\mathrm{z}$ direction) magnetic field. By measuring the trajectory of the charged particles produced in the collisions, the charges, positions and momenta of all produced charged particles is determined. A radius of $1 \mathrm{~m}$ is allocated to the vertex and tracking systems in order to achieve this objective with sufficient accuracy.

The size of the calorimetry is dictated by the characteristic distance which is needed to initiate an interaction, either electromagnetic (ECAL) or hadronic (HCAL). These sizes are:

$$
\begin{aligned}
& \left(X_{o}\right)_{P b}=0.56 \mathrm{~cm} \\
& \left(\lambda_{o}\right)_{\mathrm{Fe}}=16.8 \mathrm{~cm}
\end{aligned}
$$

The showers in ECAL are fully developed and contained in about 20 radiation lengths, $X_{o}$. Allowing space for shower sampling and readout, $20 \mathrm{~cm}$ in depth is used for ECAL. The HCAL follows in depth with $1.5 \mathrm{~m}$ of steel or 8.9 nuclear absorption lengths, $\lambda_{o}$. The focus in angular coverage of all the subsystems is in the “barrel” region, $|\eta|<1.5$. Coverage by the vertex, tracking and ECAL subsystems goes only to $|\eta|<2.5$.

In the region $2.5<|\eta|<5.0$ the radiation field at the LHC precludes all but very radiation resistant calorimetry. This forward calorimeter region is not shown explicitly, but is thought to reside at $\mathrm{z}=10 \mathrm{~m}$, a large distance which reduces the radiation dose. If it were stationed at $\mathrm{z} \sim 3.2 \mathrm{~m}$, the dose would be $\sim 9.8$ times larger with a substantial added radiation resistance required of the specific technology chosen for the calorimetry.

The high rates of background processes, as shown in Fig. 3, imply that the leptons must be measured in robust and redundant systems. For example muons are measured in the tracking systems and then again in redundant specialized muon systems which have lower rates of fairly pure muons because almost all other SM particles have been absorbed in the thick calorimeters. Electrons are also measured redundantly, in this case using the tracking systems and the electromagnetic calorimeters. In this way, the generic detector allows for clean lepton identification and measurement even though the cross sections of most interest, such as Higgs decays, are $\sim$ fb or smaller, while the inelastic backgrounds are $\sim \mathrm{mb}$, a factor of at least $10^{12}$ larger.

\section{The Generic Vertex Subsystem}

The subsystem at smallest transverse radius, $r$, is the vertex subsystem. It consists of pixels of silicon deployed from radius $10 \mathrm{~cm}$ to $20 \mathrm{~cm}$ in three layers in the generic 
model. The vertex detector is used to efficiently find and identify the secondary decay vertices of the heavy quarks and leptons as shown in Table 1 . The lifetimes of these particles set the scale for the pixel size.

$$
\begin{aligned}
& (c \tau)_{\tau}=87 \mu m \\
& (c \tau)_{b} \sim 475 \mu \mathrm{m} \\
& (c \tau)_{c} \sim(123,312) \mu m \quad\left(D^{o}, D^{ \pm}\right)
\end{aligned}
$$

Assuming a pixel size of $\delta z=\delta s=200 \mu \mathrm{m}$, where $\mathbf{s}$ is distance in the azimuthal direction ( on a cylindrical vertex pixel layer) and $\mathrm{z}$ is along the beam direction, the vertex detector spatial resolution is sufficient to identify and measure the decay vertices. Note that analogue readout of the energy deposited in neighboring pixels yields better spatial resolution then a simple digital readout result which is the pixel width divided by $\sqrt{12}=3.46$. That factor is used to convert from the full extent of a uniform distribution to the r.m.s. of that distribution.

As an example of the utility of b vertex identification, the strong production of top pairs is a large background to the measurement of the rarer electroweakly produced $\mathrm{W}$ pairs which we wish to measure at one TeV $\mathrm{W}$ pair mass. In this case tagging of $b$ quarks is useful since it serves to indicate that the $W$ pairs arise from the copious top background in the process $p+p \rightarrow t+\bar{t} \rightarrow W^{+}+b+W^{-}+\bar{b}$.

An event at the Fermilab Tevatron is shown in Fig. 10. The Lorentz boost, $\gamma_{b}=E_{b} / M_{b}$, means that, for example, $\mathbf{b}$ decays are separated from the primary vertex by distances measured in millimeters, $\gamma_{b}\left(c \tau_{b}\right)$.
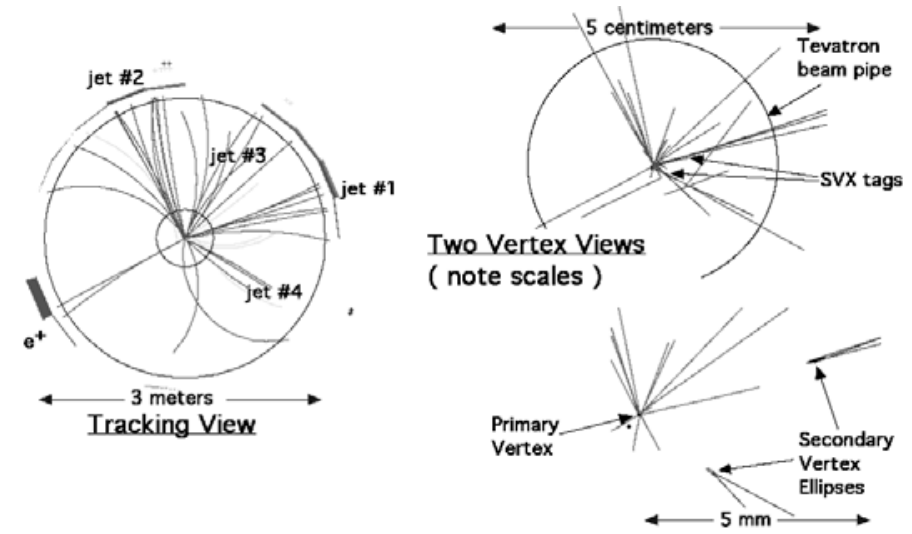

Figure 10: An event in the CDF detector with two secondary vertices coming from decays of $B$ mesons, which are seen to be separated from the primary production vertex by transverse distances of order $\mathbf{m m}$.

The probability for a pixel to be struck by a charged pion in a single bunch crossing is: 


$$
P_{p i x} \sim D_{c} N_{I}(\delta z / r)(\delta s / 2 \pi r)
$$

With a density, $D_{c}$, of 6 charged pions per unit of $\mathbf{y}$, and $N_{I}=25$ inelastic events per bunch crossing at full luminosity, the pixel occupation probability at the innermost layer at a radius $r=10 \mathrm{~cm}$ is $\sim \mathbf{0 . 0 0 0 0 9 5}$. The low occupation probability is achieved at a cost of the independent readout of many silicon pixels. Roughly, for 3 layers extending over $|z|<80 \mathrm{~cm}$ there are 8000 pixels per layer in $\mathrm{z}$ and 4700 pixels in azimuth for a total of $\mathbf{1 1 0}$ million pixels covering $|\eta|<2.5$. The sparse occupation of the pixel system makes it an ideal location to start finding track patterns. Indeed, the required high rates at the LHC were what caused ATLAS and CMS to adopt pixel detectors, which was the first time pixel detectors were deployed at hadron colliders.

Clearly, there is a tradeoff in radius between the number of pixels and the radiation field. If the vertex system could start at a radius of $5 \mathrm{~cm}$ then the number of pixels would be reduced to 28 million and the radiation would be increased fourfold due to the $r^{2}$ behavior of the number of pixels and the $1 / \mathbf{r}^{2}$ behavior of the dose. A smaller radius also reduces the position error made in extrapolating from the inner pixel layer to the actual decay vertex because of multiple scattering in the beam vacuum pipe and the detectors which causes angular track errors.

The radiation field can be approximately evaluated using the number of charged tracks leaving ionization in the silicon detectors, ignoring increases due either to secondary interactions of those tracks, photon conversion, or their multiple passage through the detectors due to particles being curled up in the magnetic field.

$$
(\text { Dose })_{\text {pix }} \sim \sigma_{I} L T D_{c}\left(1 / 2 \pi r^{2}\right) d E_{\text {ion }} / d\left(\rho_{S i} \delta r\right)
$$

The dose is the energy deposited per weight and $1 \mathrm{Mrad}=6.2 \times 10^{10} \mathrm{GeV} / \mathrm{gm}$. The number of inelastic events per second is $\sigma_{I} L=10^{9} \mathrm{~Hz}$ and the exposure time is taken to be a "year" $\mathrm{T}=1 \mathrm{yr}$ defined to be $10^{7} \mathrm{sec}$. With a density, $D_{c}$, of 6 charged particles per interaction and vertex detectors at $r=10 \mathrm{~cm}$ the charged particle fluence is $9.5 \times 10^{13} \pi^{ \pm} / \mathrm{cm}^{2}$ yr Each minimum ionizing particle at normal incidence deposits $d E_{i o n} / d\left(\rho_{S i} \delta r\right)=1.66 \mathrm{MeV} /\left(\mathbf{g m} / \mathrm{cm}^{2}\right)$ or $0.12 \mathrm{MeV}$ in a $\delta r=300 \mu \mathrm{m}$ thick detector. The estimated dose is then $2.5 \mathrm{Mrad} / \mathrm{yr}$ for the inner vertex detectors.

The results of a detailed Monte Carlo model are shown in Fig. 11. A Grey is equal to 100 rads. Note that the dose is about $5 \mathrm{Mrad} / \mathrm{yr}$ at a radius of $10 \mathrm{~cm}$, is roughly flat in $\mathrm{z}$ and falls roughly as the square of the radius as expected from the simple estimate made above. 


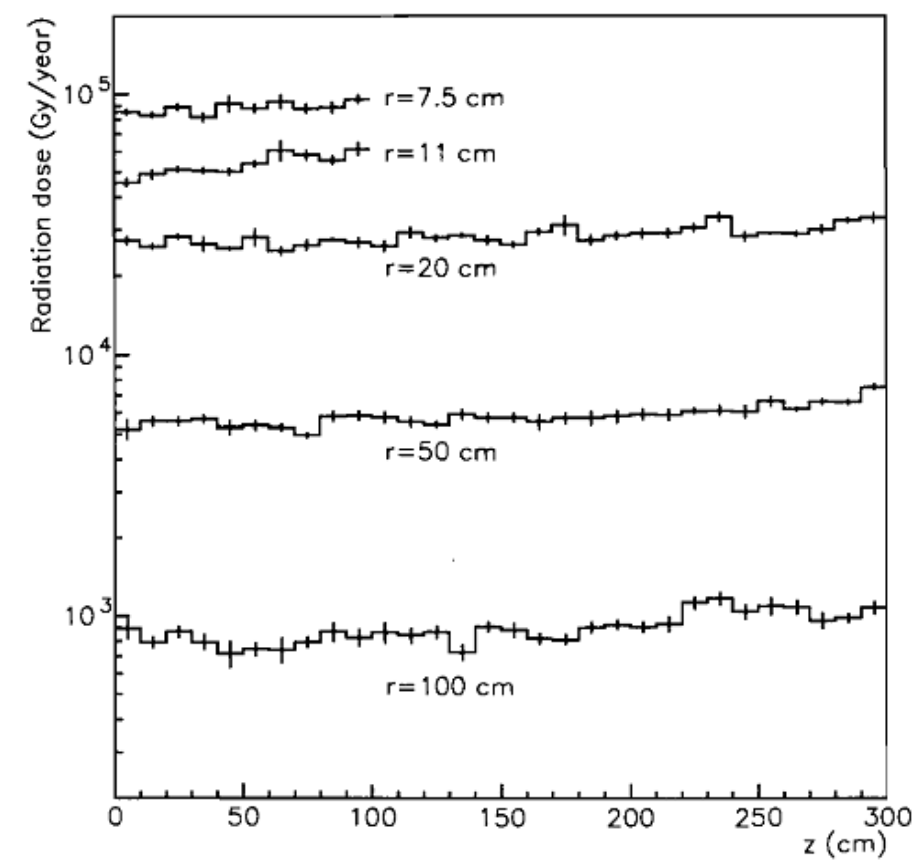

Figure 11: Radiation dose in a tracking system as a function of $\mathrm{z}$ at various radii [4].

The pixel vertex detectors could be deployed as a barrel covering $|y|<1.5$ and an endcap covering the region $1.5<|y|<2.5$. In that case the barrel inner radius of $10 \mathrm{~cm}$ would extend to $\pm 21 \mathrm{~cm}$ in $\mathrm{z}$ and the endcap at $\pm \mathrm{z}=21 \mathrm{~cm}$ would have an inner radius of only $3.4 \mathrm{~cm}$. Thus the radiation dose would be 8.6 times higher in the inner endcap in this configuration.

However, the long barrel pixel system has a problem at small angles too. The $\delta r=300 \mu \mathrm{m}$ thick detector is traversed $(1 / \sin \theta \sim 6.1,|\eta|=2.5)$ with a length of $1800 \mu \mathrm{m}$ so that the deposited charged is shared over 9 pixels which degrades the position resolution. Analogue readout of the charge sharing can recover some of this loss, but the occupation probability increases and the signal/noise ratio of each pixel decreases. In fact, both ATLAS and CMS chose the barrel/endcap geometry for these reasons.

\section{The Generic Solenoid Magnet}

The vertex and tracking subsystems are assumed to be immersed in a strong solenoid field. Roughly speaking, the current I flowing through $n$ turns/length of conductor leads to a field; $B=\mu_{o} n I$. Suppose there were conductors of size $2 \mathrm{~cm}$ in $\mathrm{z}$ all stacked by 4 in $r$ for a total of $n=200$ turns $/ \mathrm{m}$. Then to achieve a field of $5 \mathrm{~T}, 20.8$ kA of current is required. A plot of the stored energy per mass vs. the stored energy of some of the solenoids used in high energy physics is shown in Fig. 12. Clearly, LHC magnets are a step above what has been previously achieved in the Tevatron (CDF and D0) as regards the solenoids used in the detectors. 


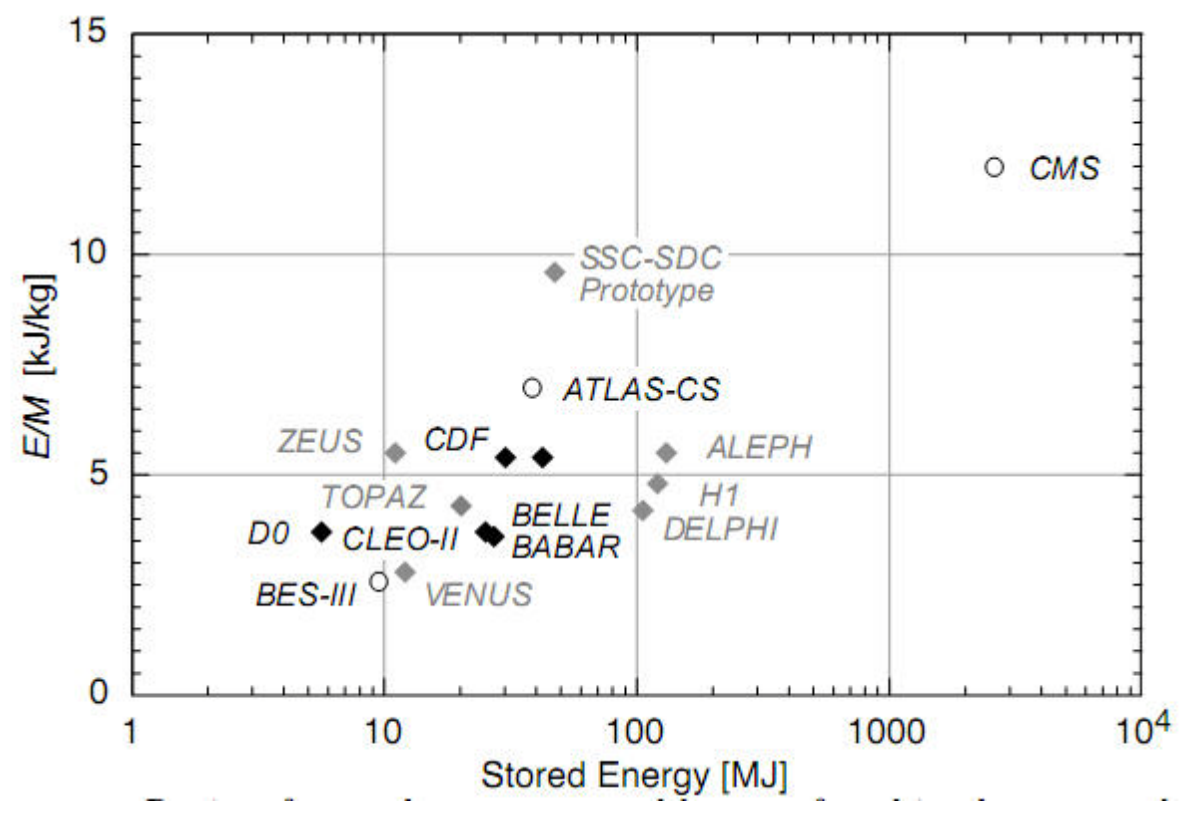

Figure 12: Energy per unit mass vs. total stored energy for a variety of solenoid magnets used in high energy physics experiments [8].

Note that in the generic detector it is just assumed that the vertex and tracking systems are immersed in a $5 \mathrm{~T}$ magnetic field. The choice of coil location is finessed; it could be just outside the tracker or just outside the calorimetry. No consensus on the location exists and therefore it is ignored here while exploring how a generic detector relates physics requirements to detector design. ATLAS and CMS have made different choices, as will be explained later.

\section{The Generic Tracker Subsystem}

The requirement that the LHC experiments decisively confront EWSB has been seen to imply a complete study of $W+W$ scattering at a mass of $\sim 1.0 \mathrm{TeV}$. In that case the transverse momentum of the $\mathrm{W}$ is $\sim 0.5 \mathrm{TeV}$ and that of the lepton for the two body $\mathrm{W}$ decay is $\sim 0.25 \mathrm{TeV}$. The radial size of the tracker is then set by the need to measure the lepton momentum well at this mass scale.

The approximate transverse momentum impulse imparted to a particle of charge e in a magnetic field $B$ in traversing a distance $r$ is;

$$
\left(\Delta P_{T}\right)_{B}=e r B=0.3 r B
$$

where the units are $\mathrm{GeV}$, Tesla and $\mathrm{m}$ and an electronic charge is assumed. The bend is in the azimuthal direction and the angle through which the particle is bent, $\Delta \phi_{B}$, is approximately the impulse divided by the transverse momentum, $\left(\Delta P_{T}\right)_{B} / P_{T}$. The error on the inverse transverse momentum is therefore; 


$$
d\left(1 / P_{T}\right)=d P_{T} / P_{T}^{2}=d\left(\Delta \phi_{B}\right) /\left(\Delta P_{T}\right)_{B} \sim d s / e^{2} B
$$

where ds is the spatial resolution in the azimuthal direction. Note that the quality factor for the momentum resolution, scales as $1 / \mathbf{r}^{2} B$ which argues for a strong magnetic field and a large radius for the cylindrical tracking subsystem.

Assuming a $5 \mathrm{~T}$ magnetic field extending over $\mathrm{r}=1 \mathrm{~m}$, the magnetic impulse is 1.5 $\mathrm{GeV}$. The bend angle is then 6 mrad for the $0.25 \mathrm{TeV}$ lepton from the $\mathrm{W}$ decay. If the tracker spatial resolution is taken by assuming the digital readout of strips of $400 \mu \mathrm{m}$ width the resolution r.m.s. is, $d s \sim 400 / \sqrt{12} \mu \mathrm{m}=115 \mu \mathrm{m}$. The momentum will then be measured to 1.9 \%. Later in this chapter, Eq.16, it is shown that a 1.2 $\%$ accuracy is needed in order not to degrade the $Z$ peak natural width to mass ratio which implies analogue readout of the tracker detector.

The momentum is measured to $10 \%$ accuracy at $1.30 \mathrm{TeV}$, which makes a sufficiently good measurement at the terascale. A smaller radius or lower field tracking system would require a more accurate measurement than the ds value quoted above if the momentum accuracy were to be maintained. Smaller strip size or analogue readout could both be used to improve the spatial resolution.

At a lower momentum scale there is another limitation to the measurement accuracy besides position resolution. The multiple scattering transverse impulse, $\left(\Delta P_{T}\right)_{M S}$, due to scattering in the tracker material compared to the magnetic field impulse sets a limit of;

$$
\begin{aligned}
& d P_{T} / P_{T} \sim\left(\Delta P_{T}\right)_{M S} /\left(\Delta P_{T}\right)_{B} \\
& \left(\Delta P_{T}\right)_{M S}=E_{s} \sqrt{\sum L_{i} / 2 X_{o}}
\end{aligned}
$$

where the scattering energy, $\mathrm{E}_{\mathrm{s}}$, is $21 \mathrm{MeV}, X_{o}$ is the radiation length, and the sum is over all material in the tracker. For a tracker plus vertex system having silicon detectors of $400 \mu \mathrm{m}$ thickness in 11 layers, 8 layers in 4 stations with small angle stereo in the tracker and 3 vertex layers, the scattering impulse is $3.2 \mathrm{MeV}$.

There are two terms in the momentum resolution, $d P_{T} / P_{T}=c P_{T} \oplus d$ which are folded in quadrature. There is a term due to measurement error, $c=0.000078 \mathrm{GeV}^{-1}$, Eq.14, and a term due to multiple scattering, $d=0.0021$, Eq.15, which limits the low momentum measurements. The crossover transverse momentum where the errors are equal is $27 \mathrm{GeV}$ in this example and the total resolution at this momentum is $0.3 \%$. Clearly, keeping the contribution due to multiple scattering low allows one to improve the momentum measurement accuracy at low momentum. By the way, only counting the detectors themselves and ignoring power leads, cooling leads and readout electronics makes this a rather optimistic estimate. 
A complete Monte Carlo model prediction for the CMS transverse momentum resolution is shown in Fig. 13. The resolution increases roughly as $P_{T}$ for momenta above $10 \mathrm{GeV}$ because of the position measurement resolution while there is no improvement at momenta below $10 \mathrm{GeV}$ due to the multiple scattering limit caused by the tracker material itself. The solenoid field chosen for the generic detector provides a fractional transverse momentum error which is independent of polar angle for $|\eta|<1$. Note that the force on a charged particle is, $\vec{F}=e(\vec{v} \times \vec{B})$, so that more forward going particles see a reduced force and a resulting deterioration in the momentum resolution.

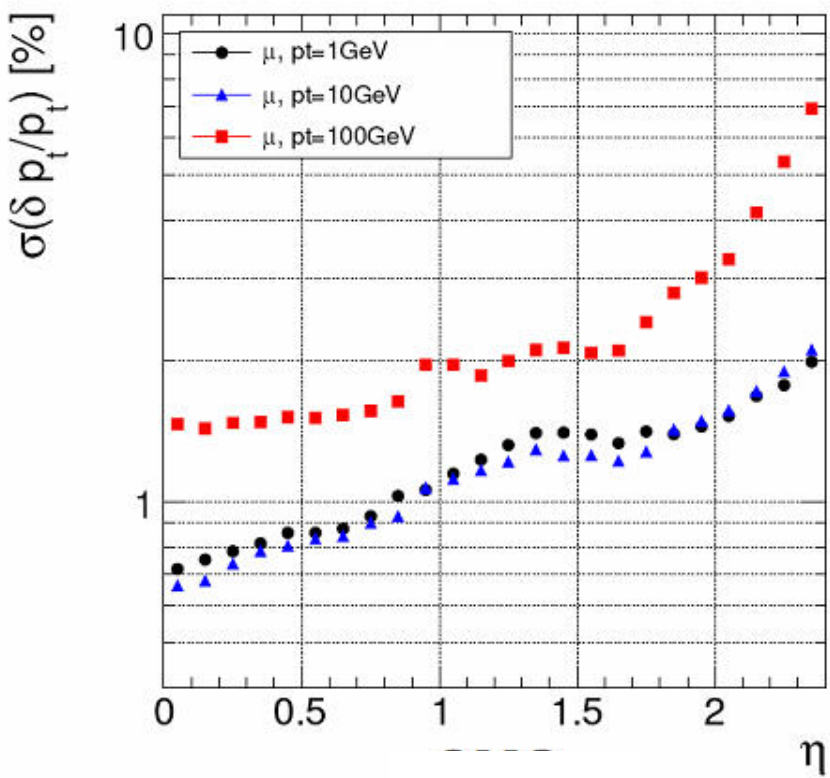

Figure 13: Transverse momentum resolution in the Monte Carlo model of the CMS detector [4].

There is additional information and improved spatial resolution available if analogue information is recorded from the tracker. For example, a measurement of the deposited energy in the silicon and the momentum as inferred from the helical trajectory results in a mass determination of the secondary particles. A plot of deposited energy, $E_{i o n}$, vs. particle momentum is shown in Fig. 14. Since $d E_{\text {ion }} / d x \sim 1 / \beta^{2}=(E / P)^{2} \rightarrow(M / P)^{2}$, the method works well at low momenta where the particles are non-relativistic. For slow particles the deposited energy goes as $M^{2}$ at fixed $P$. At high momenta all particle velocities approach $c$ and particle mass discrimination is lost. 


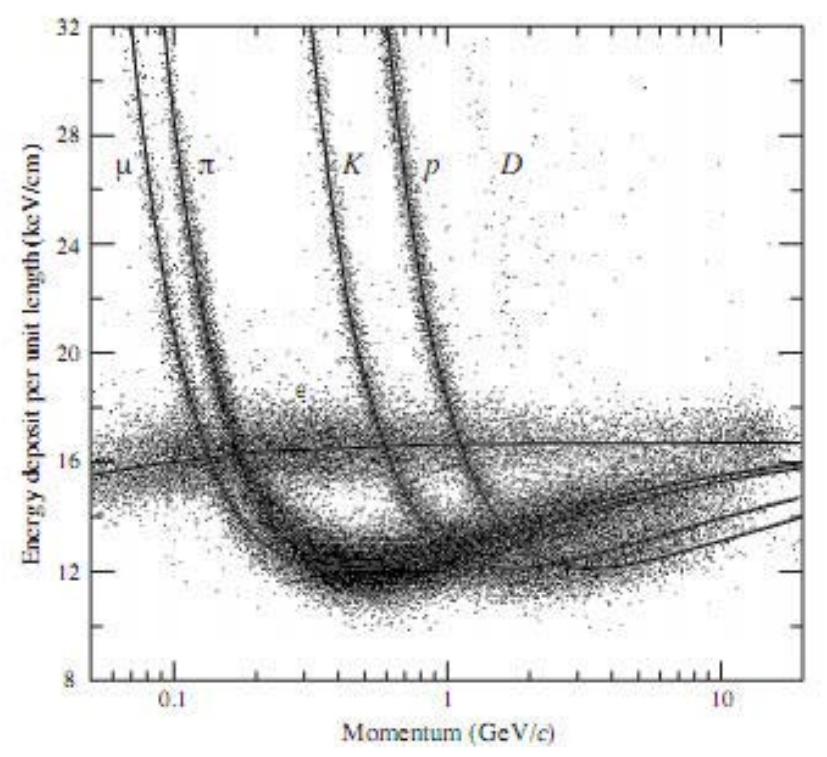

Figure 14: Deposited energy in a tracking system as a function of particle momentum for several types of particles. [8]

Most of the information provided by the tracker is contained in the measurement of the azimuthal coordinates (the "bend plane"), while the helical paths are less demanding in the $\mathrm{z}$ direction. Therefore, in order to reduce costs, the generic tracking detector is fashioned of silicon strips, $10 \mathrm{~cm}$ long in $\mathrm{z}$ and $400 \mu \mathrm{m}$ wide in $\mathrm{s}$. Each of the 4 stations has a strip layer oriented along $z$ as the long axis and a second at small angle to the first. Using Eq. 11, the probability for a strip to be occupied ranges from 0.006 to 0.00096 as the strip radius goes from 40 to $100 \mathrm{~cm}$. This level of occupation is sufficiently sparse to allow for robust track pattern recognition and trajectory fitting. The total number of strips in one layer at an average radius of 70 $\mathrm{cm}$ is $\sim 52$ in $\mathrm{z},|\mathrm{z}|<2.6 \mathrm{~m}$, times $\sim 11000$ in $\mathrm{r}$. Therefore in eight layers there are roughly 4.6 million independent strips to record.

Note that gaseous tracking detectors can also be used. A typical drift velocity is $v_{d} \sim 5 \mathrm{~cm} / \mu \mathrm{sec}$, or a drift distance of $0.12 \mathrm{~cm}$ per 25 nsec bunch crossing.

Therefore, a gaseous tracking system will likely integrate over several bunch crossings in time, thus increasing the occupancy and making the pattern recognition more difficult.

The radiation dose scales from the pixel dose as $1 / \mathbf{r}^{2}$, which means that the tracker silicon strip detectors suffer far less dose than the pixel detectors. In estimating the dose it was assumed that neutral photons are not converted to electron-positron pairs in the tracker material and that the charged pions do not interact to produce multiple charged pions. It is also assumed that particles are not bent and pass only once through a given radius. 
None of these assumptions is correct. In particular, if the transverse momentum impulse imparted to a particle is twice the transverse momentum of the particle that means it has been curled up in the field. In that case, $\left(\Delta P_{T}\right)_{B}=2\left(P_{T}\right)_{\text {loop }}=\mathrm{erB} / 2$.

Particles with transverse momentum of $0.75 \mathrm{GeV}$ never get to radii greater than the tracker radius. They are "loopers" which curl up in the tracker volume and make multiple hits in the detectors, thus increasing the dose and the occupation probability. Since the mean transverse momentum of produced pions is $\sim 0.8 \mathrm{GeV}$, Fig. 6, about one half of all charged particles produced in an inelastic collision never travel beyond the generic tracking system.

As will be mentioned below, this has negative implications for measuring the momentum carried by low energy particles in a "jet" of hadrons because they will not even reach the calorimetry. A more sophisticated use of calorimetry plus tracking, called "particle flow", is called for where the tracker is used to recover the "loopers" which are lost to the calorimetry and to potentially improve the momentum of charged tracks which are well measured in the tracker and not as well in the calorimetry.

\section{The Generic ECAL}

The generic electromagnetic calorimeter has the role of measuring the energy and position of the photons and electrons created in the LHC collisions. It is the first of the calorimeters to be struck by outgoing particles in the generic detector.

Calorimeters measure energy by initiating interactions of the incident particles and completely absorbing the resulting energy which appears as a geometrically growing "shower" of particles. As compared to the tracking and vertex systems which absorb only the tiny ionization energy deposited in them, the calorimeter readout is "destructive" in that the showering particle is totally absorbed.

The ECAL supplies a redundant measurement of the electrons. The tracking system first measures the electron momentum, charge, position and direction. The ECAL redundantly measures the electron position and energy. The electrons are important in the study of vector boson interactions because they are decay products of the $W$ and $\mathrm{Z}$. Redundancy is needed in order to be able to cleanly study the rare interactions of vector bosons using the final state electrons in a background of strongly produced neutral pions and the photons resulting from their decay.

The electrons appear as decay products of the electroweakly produced vector bosons, $Z \rightarrow e^{+}+e^{-}, W^{-} \rightarrow e^{-}+\bar{v}_{e}$. The ECAL energy resolution requirement is set by the natural width, $\Gamma_{Z}$, of the $Z$ resonance. The Breit-Wigner full width of the $Z$ resonance is irreducible and the resolution of the ECAL energy measurement should not increase the width of the di-electron mass peak beyond the irreducible scale set by the natural width. The factor 2.36 is used to convert full width at half maximum to r.m.s. 


$$
\begin{aligned}
& \Gamma_{Z}=2.5 \mathrm{GeV}, M_{Z}=91.2 \mathrm{GeV} \\
& (d E / E)_{E C A L}<\Gamma_{Z} /\left(2.36 M_{Z}\right)=1.2 \%
\end{aligned}
$$

It is customary to decompose the calorimetric energy resolution into a stochastic coefficient $a$ and a constant term $b$ and fold the two contributions in quadrature, $d E / E=a / \sqrt{E} \oplus b$. The constant term arises from the non-uniformity of the medium and the requirement is then clearly to have $b<<1 \%$. This means that the manufacturing process control of the ECAL must be very stringent.

For the stochastic coefficient, the energy of the $Z$ decay electrons is $E \sim 0.25 \mathrm{~T}$ for 1 TeV mass $Z+Z$ scattering, so that the coefficient a should be $<19 \%$. To achieve that it is important to first understand the electromagnetic showering process in better detail. The critical energy for an electron is that energy where radiative processes are comparable in probability to non-radiative processes such as ionization. In $\mathrm{Pb}$ the critical energy, $E_{c}$, is $7.3 \mathrm{MeV}$. At that energy the electron is quite relativistic having a Lorentz boost factor of, $\gamma=14.3$. The radiation length, which sets the length scale for radiative processes, is $X_{o}=0.56 \mathrm{~cm}$ in $\mathbf{P b}$.

The development of a shower is parameterized [8] using the depth, $L$, in radiation length units, $t=L / X_{o}$ and energy in critical energy units, $y=E / E_{c}$. The incident energy is $E_{o}$ and $a \sim 1+(\ln y) / 2$. The shower development in depth is then;

$$
\begin{aligned}
& d E / d t=E_{o} b(b t)^{a-1} e^{-b t} / \Gamma(a) \\
& t_{\max }=(a-1) / b, b \sim 0.5
\end{aligned}
$$

A typical shower profile for $30 \mathrm{GeV}$ electrons is shown in Fig. 15. There is a rapid rise due to the geometric shower growth. Because the energy of the electrons and photons in the shower is shared over more particles as the shower grows with depth, the average particle energy falls with depth until a point comes where radiative shower growth stops, at the "shower maximum" at a depth $t_{\max }$ where the average particle energy is near the critical, energy. The shower then dies out at greater depths by loosing energy due to ionization and photoelectric absorption. This behavior is due to the geometric behavior of the shower.

$$
\begin{aligned}
& N_{s} \sim\left(E / E_{c}\right) \sim 2^{t_{\max }} \\
& t_{\max } \sim \ln \left(E / E_{c}\right)
\end{aligned}
$$




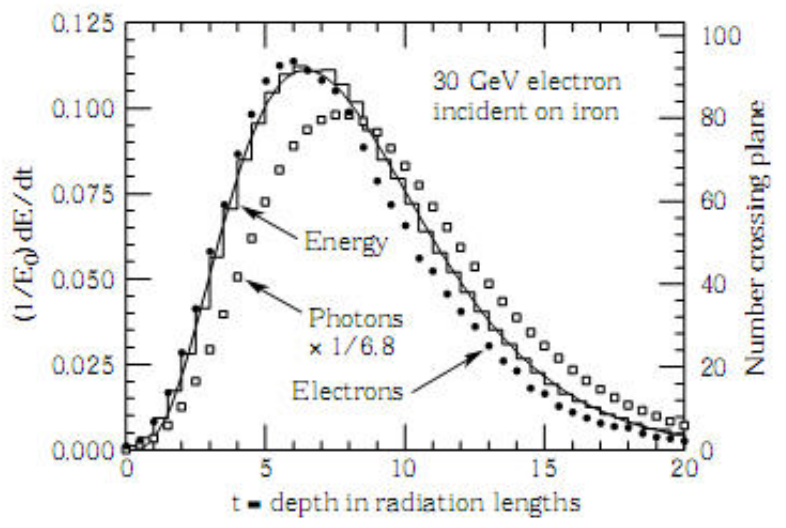

Figure 15: Longitudinal electromagnetic shower development for $30 \mathrm{GeV}$ electrons incident on iron and showering into photons and electrons. [8]

For the tracker, Eq. 14, the fractional momentum resolution scales as $1 / r^{2}$ and increases linearly with the momentum. For calorimetry the needed depth scales logarithmically with the energy. The fractional energy error also improves or stays constant with energy. Therefore, calorimetry tends to do the best job at high momenta while tracking excels at lower momenta.

As seen in the figure, the shower is fully contained over a depth $\mathbf{t} \sim 20$. It develops rapidly, so fine sampling of the shower, $\delta t \sim 0.5$, or a total of 40 depth samples is called for to achieve good energy resolution. Shower maximum occurs at $t_{\max } \sim 7$ and the full width of the shower development is $\Delta t \sim 7$.

The number of shower particles is large, peaking in Fig. 15 at 100 per sample. With fine sampling and a very uniform medium the stochastic fluctuation of the number of shower particles, $N_{s}$, can largely determine the energy resolution, $d E / E \sim 1 / \sqrt{N_{s}} \sim \sqrt{E_{c} / E}$. For $\mathbf{E}=\mathbf{0 . 2 5} \mathbf{T e V}$, the number for Pb is 34245 with a 0.54 $\%$ fluctuation which meets the energy resolution requirement. The stochastic coefficient is estimated to be, $a=\sqrt{E_{c}}=8.5 \%$ where $\mathrm{GeV}$ energy units are used. This is near the ultimately achievable resolution and most sampling calorimeters do not have as good performance. However, only $19 \%$ is required. At lower mass scales such as $0.2 \mathrm{TeV}$ a better stochastic coefficient, $\sim 8.5 \%$ would be needed.

The ECAL provides particle identification for photons and electrons in addition to energy and position measurements, in the sense shown in Table 1. An incident hadron begins to interact over a characteristic distance, $\lambda_{o}$ which is $10.2 \mathrm{~cm}$ in $\mathbf{P b}$. Thus, the fully contained ECAL shower in $t=20$ presents only a depth of $1.1 \lambda_{o}$ to an incident hadron. This difference in the length scale of hadronic and electromagnetic interactions enables the ability to distinguish between electrons and 
photons and the hadrons. Independent readout of several depth segments of each ECAL tower improves the particle identification performance.

The transverse shower development is also important as it defines the requirement for the ECAL tower size and also provides additional particle identification capability. The Moliere radius, $r_{M}$, is the radius of a cone within which $90 \%$ of the shower energy is deposited. In $\mathrm{Pb}$ the radius is $r_{M}=1.6 \mathrm{~cm}$ and that sets the tower size. Finer tower segmentation is not called for since no new information in the dense core of the shower can reasonably be extracted. Particle identification follows from the fact that hadron showers are much wider transversely with a radius $\sim \lambda_{0}$.

In the generic detector the barrel ECAL exists at $r_{E}=1.2 \mathrm{~m}$ and $\left|z_{E}\right|<2.8 \mathrm{~m}$ covering the range $|\eta|<1.5$. The ECAL towers are defined by the Moliere radius in lead, $\delta \eta=\delta \phi \sim\left(2 r_{M}\right) / r_{E}=0.027$. The barrel has 175 towers in $\mathrm{z}$ and 236 towers in azimuth. If there are three depth segments read out independently in order to measure the shower development in depth $t$ there are a total of 124,000 ECAL readout channels in the barrel alone.

It is desirable to distinguish between the electromagnetically produced photons and the strongly produced, much more copious, neutral pions. The pions decay into two photons, with a typical opening angle of $\theta \sim m_{\pi} / P_{\pi}$. With the ECAL transverse segmentation, pion momenta of $<4.2 \mathrm{GeV}$ give resolvable showers. At higher momenta the showers are not resolved and therefore appear to be isolated photons. Better rejection requires use of a detector with finer segmentation located earlier in the shower before the shower spreads to fill the Moliere radius. Such "preshower" detectors are not explored in the generic detector example.

The probability to have an overlapping neutral pion in an ECAL tower due to event pileup is; $\left(D_{o}=3\right)\left(N_{I}=25\right)(\delta \eta)^{2} / 2 \pi=\mathbf{0 . 0 0 8 7}$ assuming a fast readout with time resolution of only one bunch crossing. Since each pion carries only $0.8 \mathrm{GeV}$ of transverse momentum, pileup on top of a $0.25 \mathrm{TeV}$ shower is not severe and does not degrade the energy resolution significantly. However, the desire to simultaneously have high speed and low noise electronics is somewhat mutually exclusive. The devil is in the details and they will be provided in subsequent chapters.

The radiation field is severe since, in contrast to the tracking, the entire particle energy is now absorbed by the medium. The radiation dose in the barrel due solely to neutral pion absorption by the ECAL medium is:

$$
(\text { Dose })_{E C A L} \sim \sigma_{I} L T D_{o}\left(1 / 2 \pi r_{E}^{2}\right)<P_{T}>/\left(\rho_{E} \Delta t X_{o}\right)
$$


With 3 neutral pions per unit of rapidity, $D_{o}$, depositing $0.8 \mathrm{GeV}$ into ECAL with density $\rho_{E}$ of Pb over a region $\Delta t X_{o}$ in depth, $\Lambda t \sim 7$, there is a dose of $\sim \mathbf{0 . 0 9 7}$ Mrad/yr in the ECAL barrel. The dose in the end cap is, however, much higher. Fundamentally that increase is due to the higher energy pions in the end cap ( at fixed transverse momentum , $E_{\gamma}=<P_{T}>/ \sin \theta$ ) and the smaller radii covered by the endcap which is located at $z_{E}$. The dose ratio between the barrel and end cap for ECAL is approximately:

$$
(\text { Dose })_{\text {endcap }} /(\text { Dose })_{\text {barrel }} \sim\left(r_{E} / z_{E}\right)^{2} / \theta^{3}
$$

For the endcap the minimum angle is at $\left|\eta_{E}\right|=2.5$, where the dose ratio is about 42 which means that the endcap ECAL has a yearly dose of about 4 Mrad. This rapid dose increase with rapidity sets the limit in the generic ECAL on the angular coverage outfitted with precision electromagnetic calorimetry.

The ECAL shower development itself is very rapid so that the speed of data collection is defined by the readout. It has been assumed to have a resolving time of less than one bunch crossing similar to the speed already quoted for the silicon tracking and vertex devices. For $\delta t=0.5$ sampling and a depth of 20 t, there would be 40 samples ganged together. With a tower having an area $3.2 \times 3.2 \mathrm{~cm}$, each sample would have about $9 \mathrm{pF}$ of source capacity if the sampling gap is $1 \mathrm{~mm}$, assuming a unity gain detector. If the entire tower is summed, $360 \mathrm{pF}$, then with $50 \Omega$ connecting readout cable the rise time of the pulse would be $18 \mathrm{nsec}$. If three longitudinal segments are read out, this reduces the signal rise time to 6 nsec. Therefore, some care is needed in ensuring that the calorimeter readout is sufficiently fast.

\section{The Generic HCAL}

The hadron calorimeter, HCAL, measures the energy of the strongly interacting quarks and gluons by absorbing the jets of particles that these fundamental particles fragment into (Table 1). In addition, the "hermetic" calorimetry measures the energy of all the secondary particles within a range of $|y|<5$ which is sufficiently complete coverage (Fig. 5) that a large missing transverse energy, MET, indicates neutrinos in the final state and thus provides both particle identification and a measurement of the neutrino transverse energy.

In principle the requirements on the HCAL are very stringent, again being set by the natural width of the $W$ boson where a di-jet resonance is now reconstructed from the quark rather than the leptonic decay of the $W$ boson. 


$$
\begin{aligned}
& W^{+} \rightarrow u+\bar{d}, c+\bar{s} \\
& \Gamma_{W} / M_{W}=2.6 \% \\
& (d E / E)_{H C A L} \sim 1.1 \%
\end{aligned}
$$

In practice, such precise energy performance, with an implied stochastic coefficient of $\sim 17.4 \%$, at an energy of $0.25 \mathrm{TeV}$ is not attainable. One reason is the small number of particles in a hadronic shower with the resulting large stochastic fluctuations in that number. The analogue of the critical energy for ECAL is the threshold energy to make more secondary pions, $E_{t h} \sim 2 m_{\pi}=\mathbf{0 . 2 8} \mathbf{G e V}$. That energy scale leads to an estimate for the number of particles in the hadronic shower of $\sim E / E_{t h}$, which implies a stochastic coefficient of $53 \%$, or $3.35 \%$ fractional energy resolution, $\mathrm{dE} / \mathrm{E}$ for $\mathrm{E}=0.25 \mathrm{TeV}$, far from the requirement set by the natural resonance width of $1.1 \%$.

One attainable goal for HCAL is rather to do a good job on isolating the strongly produced top pair reducible background to the electroweakly produced $W$ pair signal. The vertex system supplies help in removing this background by making "b tags" of events with secondary vertices for $b$ jets which can then be removed. A clean sample of top pairs with hadronic $W$ decay also allows for a measurement of the di-jet mass resolution at the $W$ mass scale and an in situ energy scale calibration point for the calorimetry, $t \rightarrow W^{+}+b \rightarrow(u+\bar{d})_{W}+b$.

The total depth of the HCAL need not be as deep as that needed to fully contain the ECAL shower because the hadron calorimetry does not achieve the desired $1.1 \%$ precision energy measurement. A plot of the depth needed for an average $95 \%$ and 99 \% shower containment as a function of energy is shown in Fig. 16.

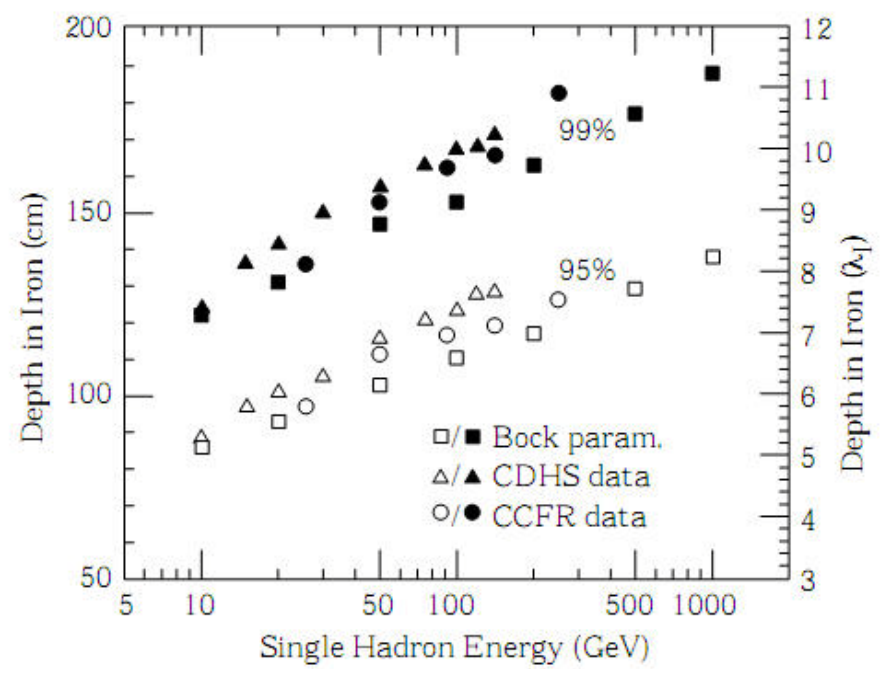

Figure 16: Depth needed for a shower energy containment of $95 \%$ and $99 \%$ as a function of hadron energy. Note the logarithmic dependence of depth on incident energy [8] 
Since the physics requirement is to fully explore $\mathrm{W}+\mathrm{W}$ scattering at $1 \mathrm{TeV}$ mass, the quarks from the $\mathrm{W}$ decay have a transverse energy of about $0.25 \mathrm{TeV}$. Asking for $99 \%$ shower containment requires about $10 \lambda_{o}$ total calorimeter depth, or about $9 \lambda_{o}$ in the HCAL. The generic HCAL has an absorber depth of $>1.5 \mathrm{~m}$ of steel or $>$ 8.9 $\lambda_{o}$ which meets this specification. In fact the "jet" of hadrons from the quark fragmentation means that the quark energy is shared over several final state hadrons, which makes the depth requirement somewhat less demanding.

Data on the longitudinal shower development for $240 \mathrm{GeV}$ pions in lead is illustrated in Fig. 17. The existence of two distance scales is clear. At each interaction in the developing shower neutral pions are produced which are then absorbed over a distance scale $X_{o}$. The produced charged pions move the energy in depth to the next interaction point with a distance scale $\lambda_{o}$ which is about twenty times larger than the radiation length for lead. To study the hadronic shower development in detail as a function of depth would require fine sampling on the scale $\delta t \sim 1$ which is roughly the scale of the sampling frequency of the data points in Fig. 17.

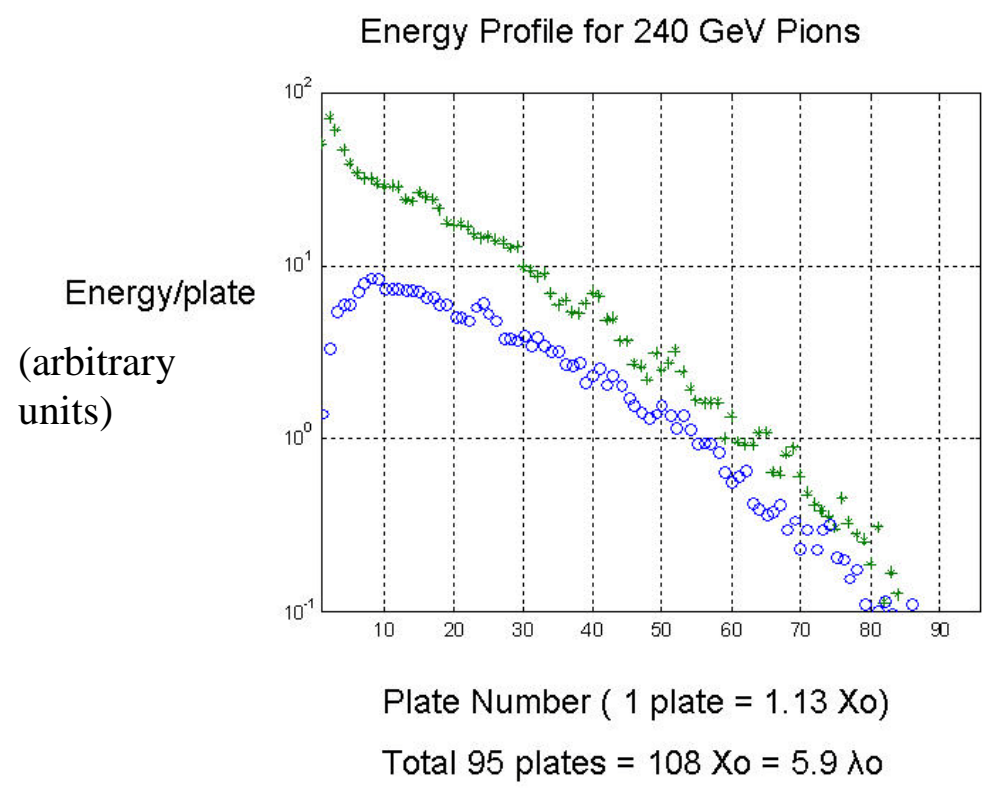

Figure 17: Data on the energy profile for charged pions with $240 \mathrm{GeV}$ energy, without subtracting the initial interaction point, 0 , and after subtraction, *.

The hadronic shower is contained transversely in a cone radius roughly of size $\sim \lambda_{0}$. Assuming that overlapping hadronic showers cannot be resolved, a size $\delta \mathrm{z}=\delta \mathrm{s}=15 \mathrm{~cm}$ is chosen in the generic HCAL. The generic HCAL begins at radius, $r_{H}=1.6 \mathrm{~m}$. The HCAL towers then subtend a rapidity and azimuthal interval; 


$$
\delta \eta=\delta \phi=0.094 \sim \lambda_{o} / r_{H}
$$

The larger transverse tower size, quoted earlier in Eq. 7, leads to a larger probability for an HCAL tower to be occupied by a charged pion, $\left(D_{c}=6\right)\left(N_{I}=25\right)(\delta \eta)^{2} / 2 \pi=\mathbf{0 . 2 1}$. The mean transverse energy in an HCAL tower is then $0.16 \mathrm{GeV}$.

The number of barrel HCAL towers in $\mathrm{z}$ is $10 \mathrm{~m} / 0.15 \mathrm{~m}=67$ and in azimuth is 67 or 4490 HCAL readout towers in the barrel. With three depth segments read out independently, there are 13,470 towers in total. Note that the showers in both the ECAL and HCAL calorimeters develop longitudinally on the scale of the radiation length so that a complete knowledge of the hadronic shower should logically have that depth segmentation. However, that level of detail has not been attempted in the LHC calorimeters.

As regards radiation, the HCAL with respect to the ECAL is at larger barrel radius but there are twice as many charged as neutral pions. The hadronic shower energy is more spatially spread out than the electromagnetic shower, Fig. 17, where the initial interaction point has a mean of one and an r.m.s. of one $\lambda_{0}$. Therefore, the full width of the deposited energy is roughly, $\Delta \lambda_{o} \sim 2 \lambda_{o}$. Taking the factors together, the dose in HCAL is about $1 / 3$ that of ECAL at the same rapidity.

However, the need to cover angles as small as $|y|=5$ arose from the requirement that the generic detector measure most of the inclusive inelastic pions and measure the forward jets from the vector boson fusion process. This requirement places a large radiation burden on the forward calorimetry. It covers the range in $|y|$ from 2.5 to 5.0. At $|y|=4$ the average pion energy is $22 \mathrm{GeV}$. Approximately 77 Watts of power is deposited by secondary particles that heat the forward calorimetry. The luminosity can be measured in the forward calorimeter with a thermometer!

At $|\eta|=5$, the polar angle is 0.75 degrees. At a location of $\mathrm{z}=10 \mathrm{~m}$ for the forward calorimetry the radius in the $(x, y)$ plane is only $13 \mathrm{~cm}$ and a "tower" with the same $\delta \eta$ as the barrel HCAL would have an extent of only $1.2 \mathrm{~cm}$ in $\mathbf{r}$ and $7.7 \mathrm{~cm}$ in azimuth, s. Clearly, such fine segmentation, given the physical size of a hadronic shower, is not very useful. Indeed, the pileup probability for a tower of transverse size $\sim \lambda_{o}$ is quite a bit higher than that for the barrel HCAL.

The radiation dose in the forward calorimetry may be roughly estimated to be ( $\mathrm{Eq}$. 19,. 20),

$$
(\text { Dose })_{\text {forward }} \sim \sigma_{I} L T D_{o}\left(1 / 2 \pi z_{F}^{2} \theta^{3}\right)<P_{T}>/\left(\rho_{\text {forward }} \Delta t X_{o}\right)
$$

Taking only the most densely deposited neutral energy to be the full radiation dose, the $\mathrm{z}$ for the calorimetry, $z_{F}$, to be $10 \mathrm{~m}$ and assuming it is made of steel, the dose is 
approximately $280 \mathrm{Mrad} / \mathrm{yr}$ at $|\mathrm{y}|=5$. The size of the dose sets the limit on the calorimetric coverage; $|y|<5$ is the limit for long term survivability of the forward calorimetry. As can be seen in Fig. 5 , there is a falloff of inclusive particle production at large $|y|$ which somewhat reduces this estimated dose.

The primary HCAL function is to measure the jets of hadrons from the "hadronization" of colored quarks and gluons. Data on the fraction of the jet energy carried by those hadrons and captured in a cone of radius $\mathbf{R}$, where $R=\sqrt{\delta \eta^{2}+\delta \phi^{2}}$ , is shown in Fig. 18. In order to capture more than $90 \%$ of the jet energy for a 100 $\mathrm{GeV}$ jet, a cone of $R>0.5$ is required. Indeed the colored quarks and gluons can always radiate a gluon by the process of "final state radiation" or FSR. The fraction

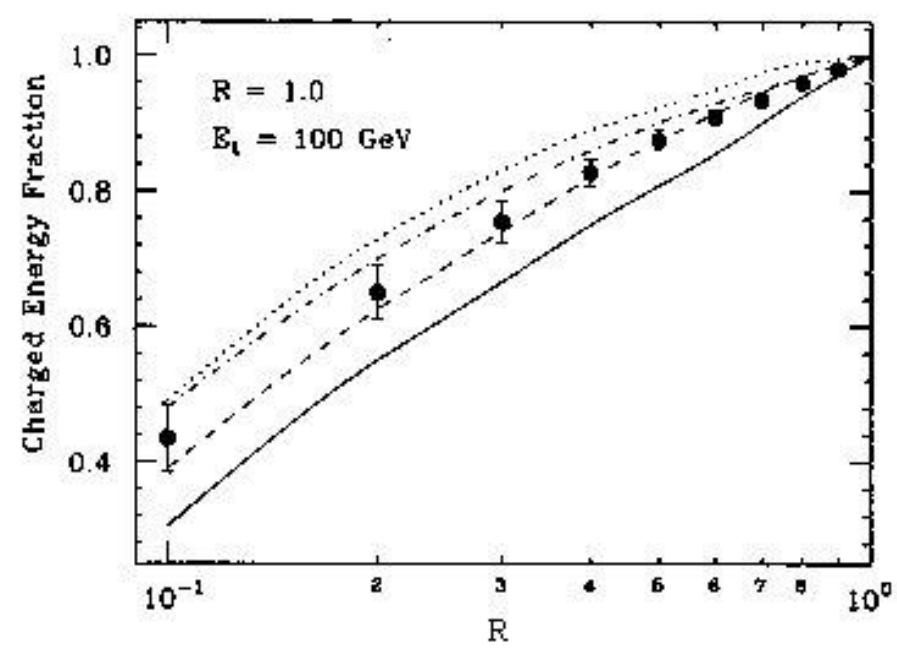

Figure 18: Fraction of the total charged particle energy for a jet which is found within a cone of radius $R$ for $100 \mathrm{GeV}$ transverse energy jets.

of jets which have radiated a fraction $\varepsilon$ of their energy outside a cone of radius $\mathbf{R}$ [12] is approximately;

$$
\text { fract } \sim\left(\alpha_{s} / \pi\right)\left[3 \log (R)+4 \log (R) \log (2 \varepsilon)+\pi^{2} / 3-7 / 4\right]
$$

where $\alpha_{s}$ is the QCD fine structure constant, numerically 0.1. Numerically, a 10 $\%$ radiation of the total jet energy outside a cone of $R=0.5$ occurs $\sim 12.5 \%$ of the time.

Clearly a limited cone size, if used to estimate the true jet energy, has a limited energy resolution due to fluctuations in the energy radiated outside the cone.

A Monte Carlo model of the $\mathrm{W}$ hadronic decay into quark pairs which then fragments into di-jets is given in Fig. 19. Clearly the FSR of gluons is an irreducible limitation in doing precision di-jet spectroscopy in the environment of a hadron collider. 
In order to reduce the problem of FSR the cone size could be increased in order to capture the radiated energy. However the pileup energy for inelastic interactions increases as the cone area. For example, a cone of radius $R=0.5$ contains $\sim 1$ unit of rapidity and azimuthal angle or $N_{I} D<P_{T}>/ 2 \pi=28.6 \mathrm{GeV}$ of pileup pions. The pileup energy will fluctuate event by event. On average there are 36 pions in the cone and if their number is stochastic, the fluctuations are 6 pions or $4.8 \mathrm{GeV}$ in transverse energy. That energy fluctuation implies a limitation on the achievable dijet mass resolution and argues against increasing the cone size much beyond $R \sim 0.5$ at full LHC luminosity.

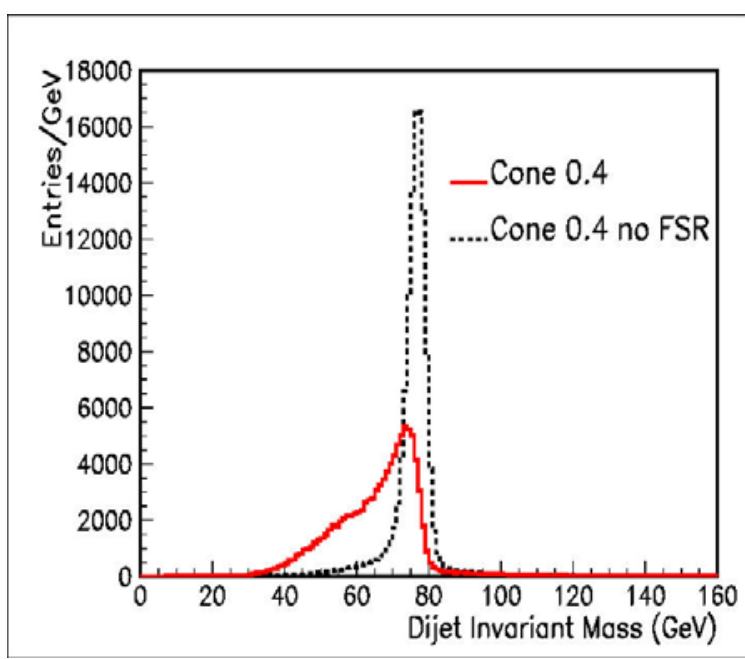

Figure 19: Monte Carlo study of $\mathrm{W}$ decay into di-jets and the resulting mass resolution both with and without FSR.

It might be imagined that setting a low threshold on the tower energies would remove the pileup issue and allow an increase in $\mathbf{R}$ so as to recover the FSR energy radiated at large $R$. However, low momentum or "soft" particles are crucial for the correct measurement of jet energies. A jet fragmenting into hadrons which carry a fraction $z$ of the jet momentum can be described by a distribution $D(z)$ parameterized as $D(z) \sim(1-z)^{a} / z$ where $\mathbf{a} \sim 5$ [13]. The fraction, $\mathbf{F}$, of the momentum of the jet contained in particles with momentum fraction $<z_{\min }$ is;

$$
F \sim 1-\left(1-z_{\min }\right)^{a+1}, z_{\min }=\left(p_{\text {had }}\right)_{\min } / P_{\text {jet }}
$$

As an example, a $50 \mathrm{GeV}$ jet has 45 of its energy carried by hadrons with momenta less than $5 \mathrm{GeV}$ and $12 \%$ carried by hadrons with momenta less than 1 $\mathrm{GeV}$. Thus the soft hadrons from the jet are easily confused with the soft pions from the pileup which then limits the achievable jet energy resolution. 
An additional issue is that the low transverse momentum charged hadrons do not even reach the calorimetry and register their energy. These "loopers" must be efficiently detected in the tracking system and the measured jet energy incremented to properly account for them.

These considerations imply that precision multijet spectroscopy is difficult at the LHC. In a more benign environment such as the proposed ILC [14], with much less pileup and no "underlying event", improved calorimetry with greatly expanded numbers of shower samples have been proposed [15] which aim to improve the calorimetric energy resolution by a factor of roughly two with respect to the LHC detectors. Using the more precise tracking measurement of low charged particle energy ("particle flow") can also improve the energy resolution of the detector overall. Another potential path to improved performance is "dual readout calorimetry" where energy measurements of the charged and neutral components of a hadronic shower are measured independently, thus allowing for different calorimetric response to these two components to be compensated for.

The calorimetric performance limitations mentioned above have implications for the reducible top pair background to electroweakly produced W pairs. At the LHC, assuming an HCAL stochastic coefficient of $60 \%$ and a constant term of $5 \%$, a top pair event where one $W$ decays leptonic has a mean MET of $52 \mathrm{GeV}$ with a MET error due to the calorimeter resolution of $\sim 17 \mathrm{GeV}$ while the $\mathrm{W}$ mass distribution from quark did-jets has a standard deviation of $\sim 5 \mathrm{GeV}$ even in the absence of the pileup.

\section{The Neutron Field}

One of the characteristics of a hadron collider detector is the existence of a large neutron field. Both the detectors and electronics must be designed being cognizant of this field. The inclusive particle flux in the small angle region is the major source of the neutrons. The results of a detailed Monte Carlo model are shown in Fig. 20.

The charged particle flux is estimated to be $\sigma_{I} L T D_{c}\left(1 / 2 \pi r_{F}^{2}\right)=9.5 \times 10^{11} \pi^{ \pm} / \mathrm{cm}^{2} y r$ at $\mathbf{r}=1 \mathrm{~m}$, in rough agreement with the results of Fig. 20. The expected steep fall off with increasing $\mathbf{r}$ is also observed. Note that at an angle of $\theta \sim 0.1 \quad(\mathbf{r}=1 \mathbf{~ m}$ at the forward calorimeter starting location of $z=10 \mathrm{~m}$ ), the pions have a mean energy of $8 \mathrm{GeV}$.

At a hadron collider there are many neutrons produced because the hadrons during the strong interaction showering process excite the calorimetric medium. That leads to neutrons when the nuclei de-excite. As a crude rule of thumb there are about 5 neutrons with a few $\mathrm{MeV}$ kinetic energy produced per $\mathrm{GeV}$ of absorbed hadrons. This then leads us to estimate the neutron flux of $3.82 \times 10^{13} \mathrm{n} /\left(\mathrm{cm}^{2} \mathrm{yr}\right)$ at a $\mathbf{1} \mathbf{~ m}$ radius, which is again in reasonable agreement with the precise results shown in Fig. 20. 

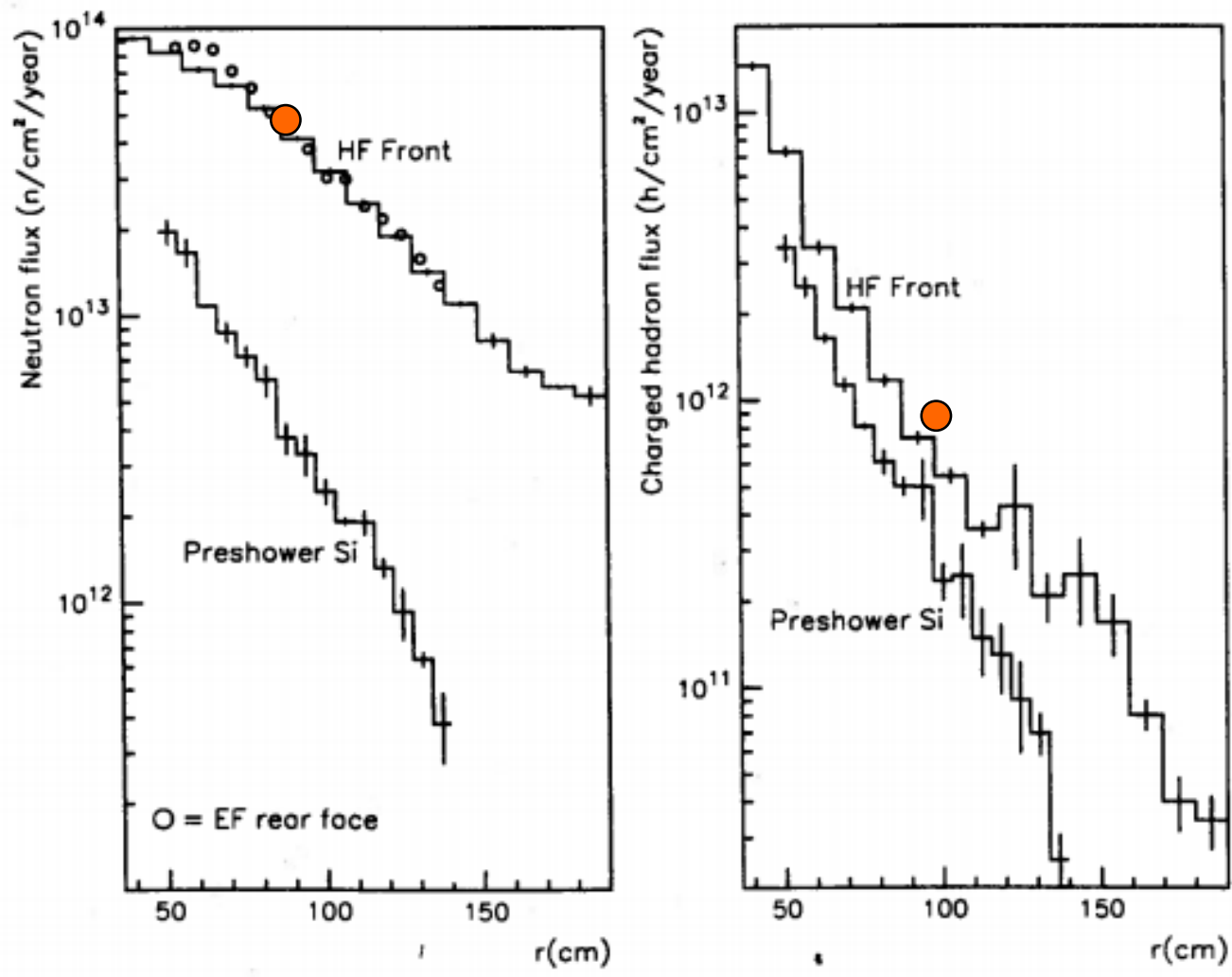

Figure 20: Charged particle flux, right, and neutron flux, left, as a function of radius for calorimetry at $\mathrm{z}=10 \mathrm{~m}[4]$.

The neutrons will elastically scatter, slow down and thermalize. In an elastic scatter off a nucleus with mass number $A$ the mean fractional energy loss for a neutron is 1/(A+1). Clearly, for heavy nuclei, the neutrons simply scatter and do not loose energy. On light nuclei, the neutrons transfer a significant energy fraction to the nucleus. The neutrons can be described as a fluid and this fluid can "flow' to all parts of the detector.

Detector design must take the neutrons into account as they can be exothermically captured and are a source of additional dose for both detectors and electronics. In fact, the design of shielding is quite important at the LHC. Complex shielding using polyethylene, borated concrete and lead are a feature of the experiments. The polyethylene is light and slows down the neutrons, the boron captures them and the lead absorbs the emitted capture photons.

If the forward calorimetry were brought closer to the interaction point in $\mathrm{z}$ to $3.2 \mathrm{~m}$ to be an extension of the end cap geometry, Fig. 9, the dose would then be higher by a factor 9.8. Moreover a tower of size $\delta r \sim \lambda_{o}$ which was chosen on the basis of hadronic shower extent would subtend an angle 0.052 radians whereas a tower of 
size $\delta \eta=0.094$ subtends an angle $\delta \theta=0.0013$ at $\eta=5$. Clearly it becomes more difficult to contain the hadronic shower in a tower with a small pileup occupancy as the rapidity increases or as the tower distance from the origin decreases.

In addition, the calorimeter is as immense neutron source and the neutrons can flow into other systems. Specifically they can make an impact on the tracking and other precision systems unless special precautions are taken.

\section{The Generic Muon System}

A major physics goal at the LHC is to explore vector boson interactions. Leptonic decays of the bosons are the favored mode since the lepton backgrounds are low and the leptons can be cleanly and redundantly measured. The muon vector momentum and position are measured first in the tracking system. The muons then deposit only ionization energy in the ECAL and HCAL while the other final state particles are almost completely absorbed. In fact the calorimetry can perform a useful muon identification role if the muons are isolated from other final state particles. Ideally, in the muon system which follows the calorimetry in depth, only muons exist along with non-interacting neutrinos.

Muons at low transverse momentum arise from the decays of inclusively produced pions and kaons. These muons can largely be removed as a background by requiring a good tracker fit to the hypothesis of no decay "kink" in the found track. For example, in the decay, $\pi \rightarrow \mu+v$ the muon transverse momentum relative to the initial pion direction is only $\sim 0.03 \mathrm{GeV}$. Therefore a $10 \mathrm{GeV}$ pion which decays in the tracker will have a 3 mrad "kink" in the full track. In discussing the generic tracker an angular resolution of $\mathbf{0 . 1 2}$ mrad was quoted. Therefore decays can be removed using the tracker goodness of trajectory fit up to a few hundred GeV. Since the probability to decay in the tracker falls with increasing momentum, pion and kaon decays are not a major problem at the high momentum scales which are of major interest to the LHC experimenters.

A second source of muons comes from heavy flavor decay, such as $b \rightarrow c+\mu+v$ which occurs much more promptly and within or prior to passage through the vertex detectors (Fig. 10). The calculated LHC cross section in leading order for production of a $b$ quark pair where one $b$ decays into a muon with transverse momentum greater than $10 \mathrm{GeV}$ is $\sigma_{\mu} \sim 60 \mu \mathrm{b}$. At the LHC design luminosity that cross section corresponds to a rate of $R_{\mu}=\sigma_{\mu} L \sim 0.6 \mathrm{MHz}$ which is small with respect to the total inelastic $\mathrm{LHC}$ rate of $1 \mathrm{GHz}$ but too large with respect to an acceptable trigger rate.

The requirements on the muon system are similar to those for the tracking system. There should be a good momentum measurement up to $\sim 0.25 \mathrm{TeV}$ for the muons. In addition, the system must produce a trigger so as to reduce the rate of background muons from the heavy flavor decays. The ionization energy loss in the 
calorimetry provides a lower momentum cutoff for the muons. The generic HCAL contains $1.5 \mathrm{~m}$ of steel, which stops all muons with a momentum less than $\sim 1.7 \mathrm{GeV}$. However, that cutoff is not sufficient to reduce the muon rate in the muon system to an acceptable level. Therefore, the muon system must measure the muon transverse momentum accurately, set a threshold value, say $10 \mathrm{GeV}$, and report it to the trigger system.

In the muon system nearly all the particles are muons, but they are of low transverse momentum and arise from heavy quark flavor decays. Since the ionization range cutoff is in energy, while the intrinsic muon rate is controlled by the transverse momentum, the rates in the forward muon systems are much larger than the rates at wide angles. Beam halo from upstream interactions in the LHC accelerator also makes the forward muon region more difficult. For these reasons, the generic muon system is thought to cover the same limited rapidity range as the tracking system, $|\mathbf{y}|<2.5$.

The steeply falling muon spectrum from heavy flavor decays means that the muon trigger system momentum resolution is very important. Poor resolution lets in a large number of triggers from lower momentum muons measured to have a higher momentum because of finite resolution, thus increasing the trigger rate. For example, if the real muon rate is characterized by an exponential falloff, $d \sigma / d P_{T \mu}=a e^{-P_{T \mu} / P_{o}}$, the observed rate is increased by a factor due to feed-down of $e^{\left(\Delta P / P_{o}\right) / 2}$ where $\Delta P$ is the muon trigger system resolution. Clearly, the trigger resolution should be rather less than the characteristic muon falloff momentum scale, $P_{o}$.

The physics process of interest is $\mathrm{W}+\mathrm{W}$ electroweak pair production. This process has a cross section evaluated at leading order of $\sim 80 \mathrm{pb}$ and top pairs provide a strongly produced reducible background with a cross section of about $680 \mathrm{pb}$ in leading order perturbation theory. The muon spectrum from the decay of the $W$ is shown in Fig. 21. The muon spectrum is strongly peaked at a transverse momentum of $\sim 40 \mathrm{GeV}$, a "Jacobean peak" arising from the kinematic behavior of the two body decay of $\mathrm{W}$ bosons which themselves have a small transverse momentum , $P_{T \mu} \sim M_{W} / 2$.

There is a complication for very high momentum muons. At the LHC their momenta are sufficiently large that radiation is an issue. The muon is a heavy electron, so that it experiences the same forces as the electron, but it has a much reduced acceleration. Radiation scales as the square of the acceleration, so that the critical energy for a muon is much larger, but still finite, compared to the critical energy for an electron.

$$
\left(E_{c}\right)_{\mu} \sim\left(E_{c}\right)_{e}\left(m_{\mu} / m_{e}\right)^{2}
$$




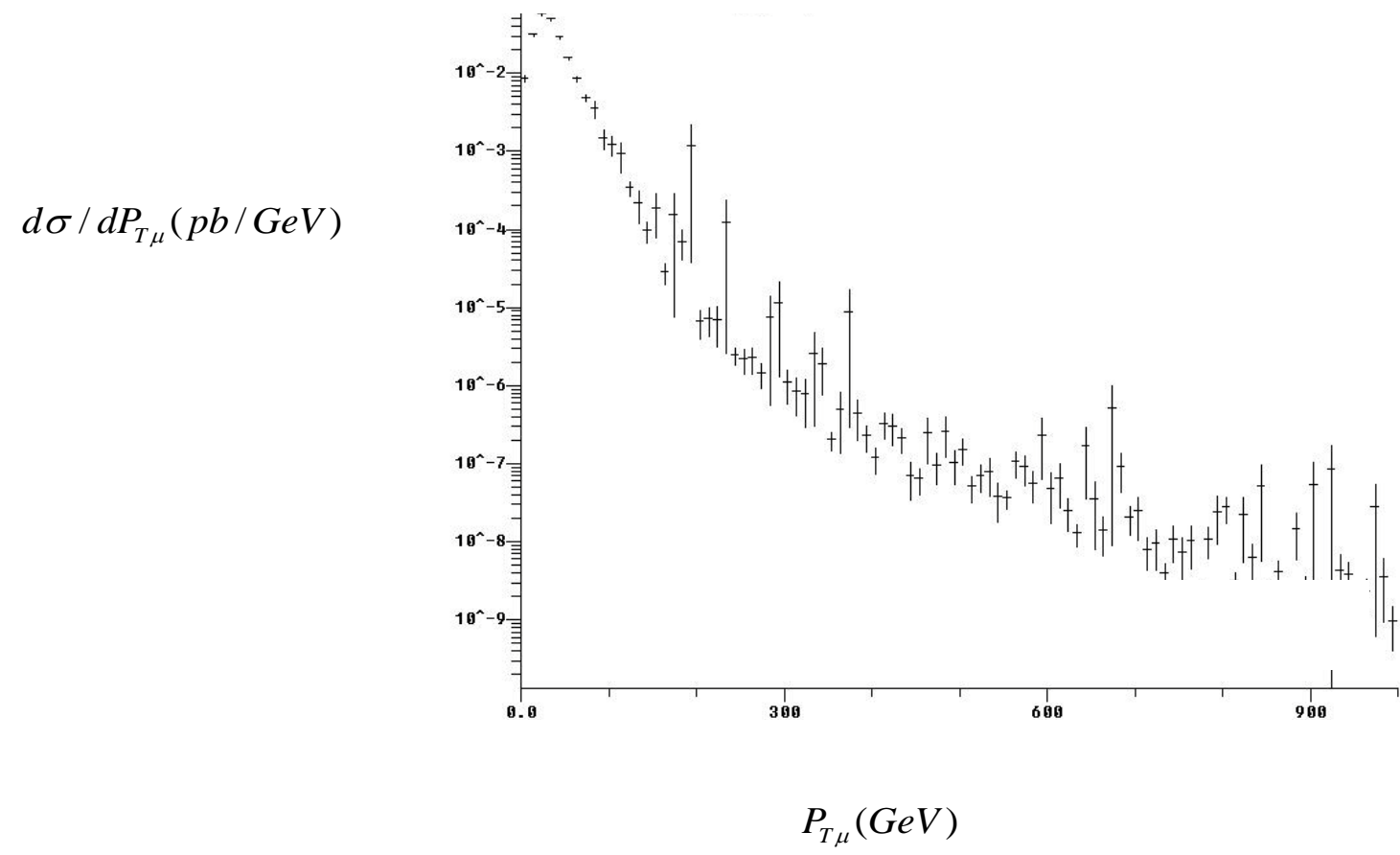

Figure 21: Muon transverse momentum spectrum in $W$ pair electroweakly produced events with no cuts on the $W$ pair mass.

For iron, the electron critical energy, 22.4 MeV scales approximately to $957 \mathrm{GeV}$ for muons. The exact energy loss for muons in iron is shown in Fig. 22. Clearly, for muons with $~ 300 \mathrm{GeV}$ momentum the radiative processes of bremsstrahlung emission and pair production are as important as ionization. This makes good tracking more difficult for the muons if the muon system tracking stations are in the solenoid flux return yoke. Note that muons in the Jacobean peak of Fig. 21 with $P_{T \mu}$ $\sim 40 \mathrm{GeV}$ have a momentum of $\sim 240 \mathrm{GeV}$ at $|\mathrm{y}|=2.5$, at the limit of muon angular coverage. Hence in the endcap region of the muon system many of the muons in the desired signal will be above the critical energy.

In general, those muons will radiate which then requires multiple measurements of their trajectories since the associated shower of collimated particles will spoil some of the measurements. A robust set of measurements can be achieved by isolating each set of measurements by interposing material of depth, $\Delta t \sim 10$, which serves to decouple each set from the others. An alternative is to operate in a large magnetic field in air which acts to sweep away the low energy showering particles.

The particle rates outside the calorimetry are sufficiently low that drift chambers with large drift distances can be used as opposed to the other subsystems in the generic detector. If the chambers are operated in "air" then the accuracy required is 
similar to that for the tracker and position resolution and alignment specifications are stringent. If the chambers are operated in the flux return yoke of the solenoid.

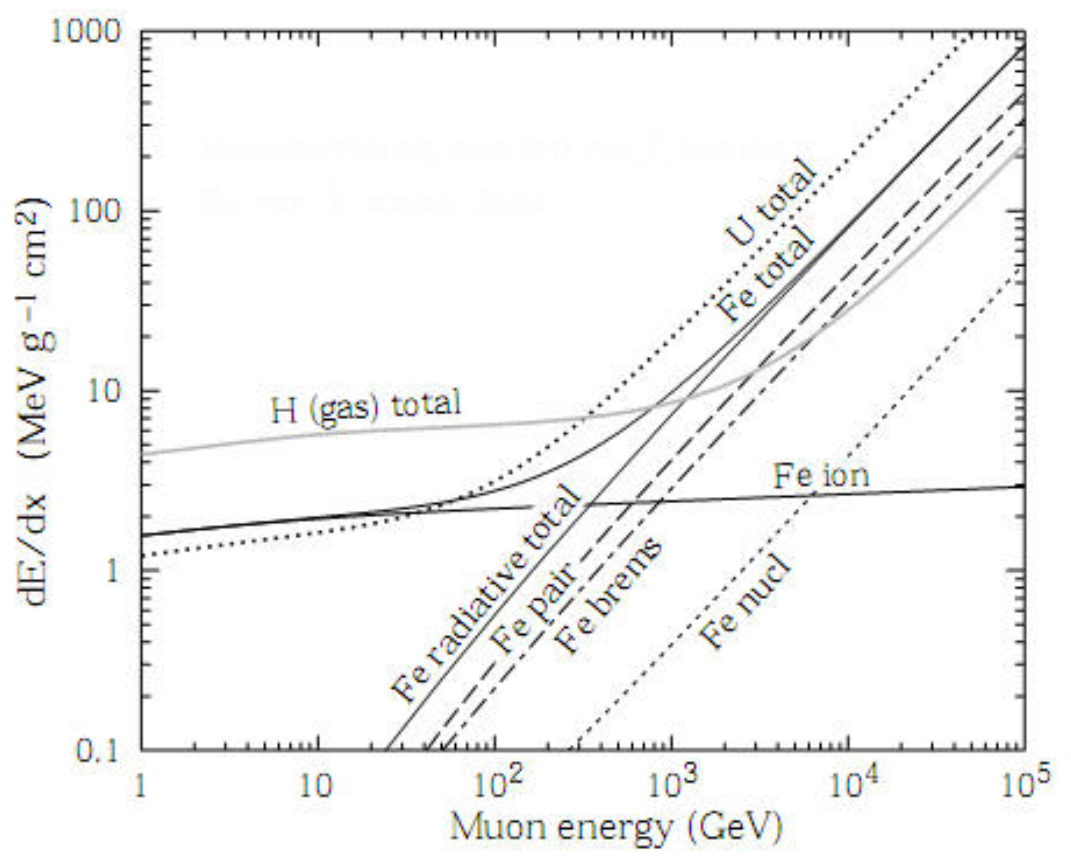

Figure 22: Energy loss of muons in iron showing ionization, pair production and bremsstrahlung contributions to the total rate of energy loss as a function of muon energy [8]

then the redundant muon momentum measurement is multiple scattering limited (Eq. 15). In that case the fractional momentum error is constant for momenta below which the alignment errors are a negligible contribution

$$
d P / P=\left(E_{s} / \sqrt{2}\right)\left[1 / e B \sqrt{L X_{o}}\right]
$$

For example with $B=2 T$ in steel with a $L=2 \mathrm{~m}$ return yoke, the muon momentum is measured to $13 \%$. The tracking provides, Eq. 14, a redundant momentum measurement with $<10 \%$ momentum error for muons with momenta $<1.3 \mathrm{TeV}$ in comparison. At still higher momenta the tracker resolution increases as $\mathbf{P}$, so the muon system will improve the tracker muon momentum resolution, if good alignment of the muon chambers can be maintained so that the measurement remains multiple scattering dominated.

For the generic detector no attempt is made to specify the muon system in detail as to detectors, magnetic field type, or medium in which the detectors are immersed. Many different choices are possible and defensible and there is no consensus on the design choices. Specific choices for ATLAS and CMS are discussed in Ref. 16. 


\section{Up the Food Chain}

The discussion so far has been about the connection between physics needs and the resultant generic detector requirements. Many of the detailed choices made by ATLAS and CMS differ. In many cases those choices have broken new ground in detector development and these aspects of the detectors and the associated design decisions will be the focus of later chapters.

The front end electronics at the LHC experiments is clocked at the r.f. bunch crossing frequency of $40 \mathrm{MHz}$. As seen in the discussion above, the radiation field is typically $1 \mathrm{Mrad} / \mathrm{yr}$ or larger which means that the electronics on the detector must be quite radiation hard. The exact front end electronics choices are very detector dependent so that a "generic" discussion is not very illuminating and has not been attempted here. The electronics must also be resistant to the large neutron background which is a characteristic feature of hadron colliders.

The inclusive rate at LHC design luminosity is $1 \mathrm{GHz}$. The number of interactions capable of being stored to permanent media is $\sim 100 \mathrm{~Hz}$. Therefore a reduction in rate of a factor of $\mathbf{1 0}$ million is needed. This is accomplished in steps. First the rate is reduced by "triggering" on leptons and jets above some transverse momentum thresholds. Imposing these thresholds on the events reduces the rate to $\sim 100 \mathrm{kHz}$. In order to not incur dead time a front end "pipeline", which stores all the data until the first stage trigger decision is made, is provided for each channel of data which is read out. There are $\sim 100$ million independent channels of information, mostly analogue, in the generic detector. Typically, after this initial decision the full event is sent from the detector front ends off the detector by means of digital optical fiber data transmission to a set of digital electronics accessible to the experimenters.

More incisive trigger decisions are then made which reduce the event rate to $\sim 100$ Hz. Even after suppression of detector elements with no hits or with signals below some low threshold, the large number of particles per event and the large number of events piling up lead to a typical event size of $1 \mathrm{Mb}$. Assuming a data taking run of 4 months per year, 1000 Petabytes/yr, or $\sim 1$ million DVDs, are stored for offline analysis. The trigger and data acquisition techniques for both ATLAS and CMS are more or less specific to the experiment and thus are not susceptible to a "generic" analysis. Again, discussions specific to the experiments appear in Ref. 16. In fact, after the first level of triggering, commercial off the shelf modules are mostly deployed. Examples are telecommunications switching networks used to assemble the full event using input from the different subsystems and "farms" of commodity PCs which are used for online high level triggering and for offline analysis. Thus, this is a good point to turn the exposition to the detailed choices made by ATLAS and CMS in their goal of decisively confronting the new physics which will be opened up at the LHC. 


\section{Acknowledgements}

The individual contributions of ATLAS and CMS authors to the chapters of Ref. 16 refer to the specific design decisions. A detailed description of the detectors and their respective full authorship lists is given in Ref. 6. All the ATLAS and CMS authors are here acknowledged for their contributions to the design, construction and installation of these two state of the art detectors.

\section{References}

1. http://en.wikipedia.org/wiki/ATLAS_experiment - and links therein http://en.wikipedia.org/wiki/Compact_Muon_Solenoid

2. http://lhc.web.cern.ch/lhc/

3. http://ab-div.web.cern.ch/ab-div/Publications/LHC-DesignReport.html

4. http://public.web.cern.ch/PUBLIC/en/LHC/CMS-en.html

5. http://atlas.web.cern.ch/Atlas/index.html

6. “The CERN Large Hadron Collider: Accelerator and Experiments”, Journal of Instrumentation,3, SO8001-SO8007, 2008

7. General-Purpose Detectors for the Large Hadron Collider, D. Froidevaux, $\mathbf{P}$. Sphicas, Ann. Rev. Nucl. Part. Sci., 56, 375, Nov. 2006

8. Review of Particle Physics, C. Amsler et al., Physics Letters B 667, 1 (2008)

9. http://en.wikipedia.org/wiki/Dark_matter and links and references therein

10. The Higgs Hunter's Guide, J. Gunion, H. Haber, S. Dawson and G. Kane, Westview Press, 2000

11. http://en.wikipedia.org/wiki/CompHEP and links and references therein

12. Jets from Quantum Chromodynamics, G. Sterman and S. Weinberg, Phys. Rev. Lett., 39, 1436 (1977)

13. "Fragmentation, Underlying Event and Jet Shapes at the Tevatron CDF)" in Hadron Collider Physics 2005, Springer, 54, 2006

14. http://www.linearcollider.org/cms/?pid=1000000 - home page for the ILC

15. http://physics.uoregon.edu/ lc/wwstudy/concepts/ - links to 4 design concepts

16. At the Leading Edge - the ATLAS and CMS LHC Experiments, World Scientific, Ed. D. Green, 2010 